\title{
Parametrized 3D models of neutrino-driven supernova explosions
}

\section{Neutrino emission asymmetries and gravitational-wave signals}

\author{
E. Müller, H.-Th. Janka, and A. Wongwathanarat
}

\author{
Max-Planck-Institut für Astrophysik, Karl-Schwarzschild-Straße 1, 85748 Garching, Germany \\ e-mail: emueller@mpa-garching.mpg.de
}

Received 30 June 2011 / Accepted 22 October 2011

\begin{abstract}
Time-dependent and direction-dependent neutrino and gravitational-wave (GW) signatures are presented for a set of three-dimensional (3D) hydrodynamic models of parametrized, neutrino-driven supernova explosions of non-rotating 15 and $20 M_{\odot}$ stars. We employed an approximate treatment of neutrino transport based on a gray spectral description and a ray-by-ray treatment of multi-dimensional effects. Owing to the excision of the high-density core of the proto-neutron star (PNS) and the use of an axis-free (Yin-Yang) overset grid, the models can be followed from the post-bounce accretion phase through the onset of the explosion into more than one second of the early cooling evolution of the PNS without imposing any symmetry restrictions and covering a full sphere. Gravitational waves and neutrino emission exhibit the generic time-dependent features already known from 2D (axi-symmetric) models. Violent non-radial hydrodynamic mass motions in the accretion layer and their interaction with the outer layers of the proto-neutron star together with anisotropic neutrino emission give rise to a GW signal with an amplitude of $\sim 5-20 \mathrm{~cm}$ in the frequency range of 100-500 Hz. The GW emission from mass motions usually reaches a maximum before the explosion sets in. After the onset of the explosion the GW signal exhibits a low-frequency modulation, in some cases describing a quasi-monotonic growth, associated with the non-spherical expansion of the explosion shock wave and the large-scale anisotropy of the escaping neutrino flow. Variations of the mass-quadrupole moment caused by convective activity inside the nascent neutron star add a high-frequency component to the GW signal during the post-explosion phase. The GW signals exhibit strong variability between the two polarizations, different explosion simulations and different observer directions, and besides common basic features do not possess any template character. The neutrino emission properties (fluxes and effective spectral temperatures) show fluctuations over the neutron star surface on spatial and temporal scales that reflect the different types of non-spherical mass motions in the supernova core, i.e., post-shock overturn flows and proto-neutron star convection. However, because very prominent, quasi-periodic sloshing motions of the shock caused by the standing accretion-shock instability are absent and the emission from different surface areas facing an observer adds up incoherently, the modulation amplitudes of the measurable neutrino luminosities and mean energies are significantly lower than predicted by 2D simulations.
\end{abstract}

Key words. stars: neutron - hydrodynamics - neutrinos - stars: massive - supernovae: general - gravitational waves

\section{Introduction}

The electromagnetic signature of core-collapse supernovae has been exploited comprehensively and thoroughly by countless observations during the past decades, providing only indirect information about the explosion mechanism, however. The up to now only recorded neutrino signal of a core-collapse supernova (SN1987A) confirmed the idea that the collapse of the core of a massive star to neutron star densities provides the necessary energy for the explosion (Baade \& Zwicky 1934). Because gravitational waves $(\mathrm{GW})$, the only other means to probe the supernova engine besides neutrinos, are yet to be detected, supernova modelers are preparing for this prospective measurement by predicting the gravitational wave signature of core-collapse supernovae with ever increasing realism (for reviews, see e.g., Kotake et al. 2006; Ott 2009; Fryer \& New 2011).

According the Einstein's theory of general relativity (GR), gravitational waves will be generated by any mass-energy distribution whose quadrupole (or higher) moment varies non-linearly with time. In core-collapse supernovae this criterion is satisfied by time-dependent rotational flattening particularly during collapse and bounce, prompt post-shock convection, non-radial flow inside the proto-neutron star and in the neutrino-heated hot bubble, the activity of the standing accretion shock instability
(SASI), asymmetric emission of neutrinos, and by asymmetries associated with the effects of magnetic fields (for a recent review see, e.g., Ott 2009, and references therein). While significant rotational deformation and dynamically relevant magnetic fields require particular progenitors that possess some (considerable) amount of rotational and magnetic energy or that must rotate fast and differentially (additional differential rotation develops during collapse) to amplify an initially weak magnetic field, all other processes are genuinely operative in any core-collapse supernova.

Obviously, an adequate modeling of these effects and an accurate prediction of the $\mathrm{GW}$ signal ultimately requires three dimensional (3D) hydrodynamic simulations covering the collapse, bounce, and post-bounce evolution (at least) until a successful launch of the explosion and including a proper treatment of neutrino transport and the relevant microphysics. However, most studies of the past thirty years were either concerned with the collapse and bounce signal only (Müller 1982; Finn \& Evans 1990; Mönchmeyer et al. 1991; Yamada \& Sato 1994; Zwerger \& Müller 1997; Rampp et al. 1998; Dimmelmeier et al. 2001, 2002; Kotake et al. 2003; Shibata 2003; Shibata \& Sekiguchi 2004; Ott et al. 2004; Cerda-Duran et al. 2005; Saijo 2005; Shibata \& Sekiguchi 2005; Kotake et al. 2006; Dimmelmeier et al. 2007; Ott et al. 2007; Dimmelmeier et al. 2008), or were 
restricted to axisymmetric (2D) models (Müller et al. 2004; Ott et al. 2006; Kotake et al. 2007; Marek et al. 2009; Murphy et al. 2009; Yakunin et al. 2010). Several authors also investigated the influence of magnetic fields on the GW signal during the collapse and early post-bounce evolution assuming axisymmetry (Kotake et al. 2004; Yamada \& Sawai 2004; Kotake et al. 2005; Obergaulinger et al. 2006a,b) and no symmetry restriction at all (Scheidegger et al. 2008, 2010). The GW signal caused by aspherical neutrino emission was first studied by Epstein (1978) and subsequently by Burrows \& Hayes (1996), Müller \& Janka (1997), and Kotake et al. (2007, 2009a,b, 2011), where the investigations by Müller \& Janka (1997) and Kotake et al. (2009b, 2011) considered also 3D, i.e., non-axisymmetric models.

Concerning 3D post-bounce models, the topic of the study presented here, Müller \& Janka (1997) were the first to analyze the GW signature of 3D non-radial flow and anisotropic neutrino emission from prompt post-bounce convection in the outer layers of a proto-neutron star during the first $30 \mathrm{~ms}$ after supernovashock formation. Because of smaller convective structures and slower overturn velocities, the GW wave amplitudes of their 3D models are more than a factor of 10 lower than those of the corresponding 2D ones, and the wave amplitudes associated with anisotropic neutrino emission are a factor of 10 higher than those caused by non-radial gas flow. Fryer (2004) and Fryer et al. (2004) presented gravitational wave signals obtained from $3 \mathrm{D}$ core-collapse simulations that extend up to $150 \mathrm{~ms}$ past bounce and were performed with a Newtonian smoothed particle hydrodynamics code coupled to a three-flavor gray fluxlimited diffusion neutrino transport scheme. Gravitational wave emission occurs owing to matter asymmetries that arise from perturbations caused by precollapse convection, core rotation, and low-mode convection in the explosion engine itself, and owing to anisotropic neutrino emission. Kotake et al. (2009b) simulated 3D mock-up models that mimic neutrino-driven explosions aided by the SASI, and computed the GW signal resulting from anisotropic neutrino emission by means of a ray-tracing method in a post-processing step. They pointed out that the gravitational waveforms of $3 \mathrm{D}$ models vary much more stochastically than those of axisymmetric ones, i.e., in 3D the GW signals do not possess any template character. However, when considering rotating models, Kotake et al. (2011) argue that the GW waveforms resulting from neutrino emission exhibit a common feature, which results from an excess of neutrino emission along the spin axis due to the growth of spiral SASI modes. Scheidegger et al. (2008) simulated the collapse of two rotating and magnetized cores in 3D until several $10 \mathrm{~ms}$ past bounce, applying a parametrized deleptonization scheme (Liebendörfer 2005) that is a good approximation until a few milliseconds past bounce. Scheidegger et al. (2010) extended their study by systematically investigating the effects of the equation of state, the initial rotation rate, and both the initial magnetic field strength and configuration on the GW signature. They also simulated three representative models until $\sim 200 \mathrm{~ms}$ of post-bounce accretion (no development of explosions) incorporating a treatment for neutrino transport based on a partial implementation of the isotropic diffusion source approximation (Liebendörfer et al. 2009).

In the following we present the GW analysis of a set of parametrized 3D models of neutrino-powered supernova explosions covering the evolution from core bounce until $\sim 1.4 \mathrm{~s}$ later, where the high-density inner core of the proto-neutron star (PNS) is excised and replaced by a time-dependent boundary condition and a central point mass. The neutrino transport is treated by an approximate solver based on a ray-by-ray treatment of the multi-dimensional effects (Scheck et al. 2006). Because we analyze the GW signal arising from both non-radial mass flow and anisotropic neutrino emission, we discuss the neutrino emission of these 3D models as well, and particularly address its multidimensional properties, some of which have previously been investigated in 2D by Janka \& Mönchmeyer (1989a,b), Ott et al. (2008), Kotake et al. (2009a), Marek \& Janka (2009), Marek et al. (2009), and Brandt et al. (2011).

Based on 2D simulations of rotational core-collapse, Janka \& Mönchmeyer (1989a,b) pointed out that the energy output in neutrinos seen by an observer may vary as much as a factor of 3 with his inclination angle relative to the rotation axis, while for the neutrino energy much smaller angular variations are to be expected. Marek et al. (2009) and Marek \& Janka (2009) found that neutrino luminosities and mean energies show quasi-periodic time variability in their $2 \mathrm{D}$ simulations of nonrotating and slowly rotating $15 M_{\odot}$ progenitors covering up to $\sim 700 \mathrm{~ms}$ past bounce. Caused by the expansion and contraction of the shock in the course of SASI oscillations, the level of the fluctuations is $\$ 50 \%$ for the luminosities and roughly $1 \mathrm{MeV}$ for the mean neutrino energies. The luminosity fluctuations are somewhat bigger for $v_{\mathrm{e}}$ and $\bar{v}_{\mathrm{e}}$ than for heavy-lepton neutrinos. The neutrino quantities also depend on polar angle as a consequence of the preference of the SASI motions along the symmetry axis of the $2 \mathrm{D}$ models. Additional short-wavelength spatial variations of the average radiated energies and of the neutrino fluxes are caused by local downdrafts of accreted matter. This is in accordance with the findings of Müller \& Janka (1997), who inferred from a post-processing analysis of the neutrino emission anisotropy that features in the GW signal of their 2D models of convection in the hot-bubble region are well-correlated with structures in the neutrino signal. The features are associated with sinking and rising lumps of matter and with temporal variations of aspherical accretion flows toward the proto-neutron star. Kotake et al. (2009a) computed neutrino anisotropies with a ray-tracing scheme by post-processing their 2D SASI models and derived analytical expressions for evaluating GW signals for neutrinos in 3D models, too. A generalization of these expressions will be presented below. Brandt et al. (2011) performed 2D multi-group, multi-angle neutrino transport simulations for both a non-rotating and rapidly rotating $20 M_{\odot}$ model extending $\sim 400 \mathrm{~ms}$ beyond bounce. Their simulations predict that the neutrino radiation fields vary much less with angle than the matter quantities in the region of net neutrino heating because most neutrinos are emitted from deeper radiative regions and because the neutrino energy density combines the specific intensities as integrals over sources at many angles and depths. The rapidly rotating model exhibits strong, flavor-dependent asymmetries in both peak neutrino flux and light curves, the peak flux and decline rate having pole-equator ratios $\lesssim 3$ and $\lesssim 2$, respectively. Brandt et al. (2011) also provide estimates of the detectability of neutrino fluctuations in IceCube and Super-Kamiokande as previously done by Lund et al. (2010) on the basis of the Marek et al. (2009) non-rotating models.

The paper is organized as follows: in Sect. 2 we discuss the numerical methods, the input physics, and the properties of the progenitor models and the set of 3D simulations that we analyzed. Section 3 contains a description of the formalism we used to extract the observable neutrino signal of our 3D models, and a discussion of some of its properties relevant for the corresponding GW signal. In Sect. 4 we give the formalism necessary to calculate the GW signature of 3D non-radial flow and anisotropic neutrino emission, and discuss the GW signature of the investigated 3D models. Finally, in Sect. 5 we summarize our results and discuss shortcomings and possible implications of our study. 


\section{Model setup}

\subsection{Code and computational grid}

The 3D supernova models we analyzed for their neutrino and GW signature have been simulated with the explicit finitevolume, Eulerian, multi-fluid hydrodynamics code Prometheus (Fryxell et al. 1991; Müller et al. 1991a,b). This code integrates the multidimensional hydrodynamic equations using the dimensional splitting method of Strang (1968), the piecewise parabolic method of Collela \& Woodward (1984), and a Riemann solver for real gases proposed by Colella \& Glaz (1985). Inside grid cells with strong grid-aligned shocks fluxes computed from the Riemann solver are replaced by the AUSM+ fluxes of Liou (1996) in order to prevent odd-even decoupling (Quirk 1994). Nuclear species are advected using the consistent multi-fluid advection (CMA) scheme of Plewa \& Müller (1999).

The simulation code employs an axis-free overlapping "Yin-Yang" grid (Kageyama \& Sato 2004) in spherical polar coordinates, which was recently implemented into PromETHEus, for spatial discretization (Wongwathanarat et al. 2010a). The YinYang grid relaxes the CFL-timestep condition and avoids numerical artifacts near the polar axis. Concerning the ray-by-ray neutrino transport no special procedure needs to be applied for the Yin-Yang grid. The (scalar) quantities involved in the transport algorithm are computed on both the Yin and Yang grid and are then linearly interpolated as any other scalar quantity.

The grid consists of $400(r) \times 47(\theta) \times 137(\phi) \times 2$ cells corresponding to an angular resolution of $2^{\circ}$ and covers the full $4 \pi$ solid angle. The radial grid has an equidistant spacing of $0.3 \mathrm{~km}$ from the inner grid boundary out to $r=80 \mathrm{~km}$ (models W15 and N20; see Table 1) or $115 \mathrm{~km}$ (model L15; see Table 1), respectively. Beyond this radius the radial grid is logarithmically spaced. The outer radial grid boundary $R_{\mathrm{ob}}$ is at $18000 \mathrm{~km}$, which is sufficiently far out to prevent the supernova shock from leaving the computational domain during the simulated epoch. This radial resolution suffices the requirement that there are always more than 15 radial zones per decade in density.

A central region, the dense inner core of the proto-neutron star (PNS) at $\rho \gtrsim 10^{12 \ldots 13} \mathrm{~g} \mathrm{~cm}^{-3}$, is excised from the computational domain and replaced by an inner time-dependent radial boundary condition and a point mass at the coordinate origin. The radius of the inner radial boundary shrinks according to Eq. (13) of Scheck et al. (2008). For the W15 and N20 models the initial and final (asymptotic) boundary radii are $R_{\mathrm{ib}}^{\mathrm{i}}=65 \mathrm{~km}$ and $R_{\mathrm{ib}}^{\mathrm{f}}=15 \mathrm{~km}$, respectively. For the L15 models the corresponding radii are $82 \mathrm{~km}$ and $25 \mathrm{~km}$, accounting for a less extreme contraction of the neutron star within the simulation time. The timescale for the contraction is $t_{\mathrm{ib}}=1 \mathrm{~s}$ for all models. This choice of parameters implies $R_{\mathrm{ib}} \approx 19 \mathrm{~km}$ at $1.3 \mathrm{~s}$ for models W15 and N20, and $R_{\mathrm{ib}} \approx 30 \mathrm{~km}$ at $1.4 \mathrm{~s}$ for the L15 models. Hydrostatic equilibrium is assumed at the inner radial grid boundary $R_{\mathrm{ib}}$, which is thus a Lagrangian (co-moving) position, while a free outflow boundary condition is employed at the outer radial grid boundary (for more details, see Wongwathanarat 2011; Wongwathanarat et al. 2010a).

\subsection{Input physics}

Self-gravity is fully taken into account by solving Poisson's equation in integral form using an expansion into spherical harmonics as in Müller \& Steinmetz (1995). The monopole term of the potential is corrected for general relativistic effects as described in Scheck et al. (2006) and Arcones et al. (2007). The
Table 1. Some properties of the analyzed 3D models.

\begin{tabular}{lccccc}
\hline \hline Model & $\begin{array}{c}M_{\mathrm{PS}} \\
{\left[M_{\odot}\right]}\end{array}$ & $\begin{array}{c}M_{\text {NS }} \\
{\left[M_{\odot}\right]}\end{array}$ & $\begin{array}{c}t_{\exp } \\
{[\mathrm{ms}]}\end{array}$ & $\begin{array}{c}E_{\exp } \\
{[\mathrm{B}]}\end{array}$ & $\begin{array}{c}t_{\mathrm{f}} \\
{[\mathrm{s}]}\end{array}$ \\
\hline $\mathrm{W} 15-2$ & 15 & 1.37 & 248 & 1.13 & 1.3 \\
$\mathrm{~W} 15-4$ & 15 & 1.38 & 272 & 0.94 & 1.3 \\
L15-2 & 15 & 1.51 & 381 & 1.74 & 1.4 \\
L15-3 & 15 & 1.62 & 477 & 0.84 & 1.4 \\
$\mathrm{~N} 20-2$ & 20 & 1.28 & 265 & 3.12 & 1.3 \\
\hline
\end{tabular}

Notes. Included: the mass of the progenitor star $M_{\mathrm{PS}}$, the mass of the neutron star $M_{\mathrm{NS}}$, the time of explosion $t_{\mathrm{exp}}$, and the explosion energy $E_{\exp }$ at the time $t_{\mathrm{f}}$ after bounce when we stopped the simulation. Note that $1 \mathrm{~B}=1$ bethe $=10^{51} \mathrm{erg}$.

cooling of the PNS is prescribed by neutrino emission properties (luminosities and mean neutrino energies) as boundary condition at the inner radial grid boundary (for details, see Scheck et al. 2006). The contraction of the PNS is mimicked by the movement of the inner radial grid boundary (see Sect. 2.1). "Ray-by-ray" neutrino transport and neutrino-matter interactions are approximated as in Scheck et al. (2006) by radial integration of the one-dimensional (spherical), gray transport equation for all angular grid directions $(\theta, \phi)$ independently. This approach allows for angular variations of the neutrino fluxes. The tabulated equation of state (EoS) of Janka \& Müller (1996) is used to describe the stellar fluid. It includes arbitrarily degenerate and arbitrarily relativistic electrons and positrons, photons, and four predefined nuclear species (n, p, $\alpha$, and a representative Fe-group nucleus) in nuclear statistical equilibrium.

\subsection{Models}

We have analyzed a set of 3D simulations (Wongwathanarat 2011; Wongwathanarat et al. 2010b, and in prep.) based on two $15 M_{\odot}$ progenitor models (W15 and L15), and a $20 M_{\odot}$ progenitor model (N20). The W15 model is obtained from the non-rotating $15 M_{\odot}$ progenitor s15s7b2 of Woosley \& Weaver (1995), the L15 model from a star evolved by Limongi et al. (2000), and the N20 model from a SN 1987A progenitor of Shigeyama \& Nomoto (1990). The progenitor models were evolved through collapse to $15 \mathrm{~ms}$ after bounce with the Prometheus-Vertex code in one dimension (Marek \& Buras, priv. comm.) providing the initial models for the 3D simulations. To break the spherical symmetry of the initial models, random seed perturbations of $0.1 \%$ amplitude are imposed on the radial velocity $\left(v_{r}\right)$ field at the beginning of the $3 \mathrm{D}$ simulations. Explosions are initiated by neutrino heating at a rate that depends on suitable values of the neutrino luminosities imposed at the lower boundary chosen such that the desired value of the explosion energy is obtained. The evolution is followed until $1.3 \dot{\mathrm{s}}$ after bounce for the W15 and N20 progenitor models, while the L15 models are simulated until 1.4 s post-bounce. The GW analysis presented below comprises five models (see Table 1), where models W15-2 and W15-4 differ only by the initial seed perturbations. The explosion energies, $E_{\text {exp }}$, given in Table 1 are instantaneous values at the end of the simulations $\left(t=t_{\mathrm{f}}\right)$, adding up the total energies (kinetic + internal + gravitational) in all zones where the sum of these energies is positive. The explosion time, $t_{\text {exp }}$, is defined as the time when this sum exceeds a value of $10^{48} \mathrm{erg}$, roughly corresponding to the time when the average shock radius is 400 to $500 \mathrm{~km}$ (see, however, Pejcha \& 
Thompson 2012, for an alternative definition of the time of the onset of the explosion).

\section{Neutrino signal}

The non-radial motions caused by the SASI and convection in the neutrino-heated hot-bubble as well as by convection inside the proto-neutron star (driven by Ledoux unstable lepton gradients) give rise to a time-dependent, anisotropic emission of neutrinos of all flavors, and thus to the emission of gravitational waves (Epstein 1978; Burrows \& Hayes 1996; Müller \& Janka 1997; Kotake et al. 2007, 2009a,b), as discussed in Sect. 4. We have analyzed this emission for the 3D models discussed above (see Sect. 2.3), particularly addressing its multidimensional properties.

\subsection{Formalism}

To derive observable luminosities of an emitting source we consider an observer located at far distance $D$ from that source (see Fig. 1). According to definition the flux measured by the observer is given by the following integral at the position of the observer:

$$
F(D, t)=\oint \mathrm{d} \omega \mu I(D, \omega, t)
$$

where $\mu$ is the cosine of the angle between the direction of the radiation and the line of sight (between the observer and the center of the source), $\omega$ denotes the radiation direction at the observer's location (defined by a pair of angles), and $\mathrm{d} \omega$ is the solid angle element around the radiation direction $\omega$. The intensity $I$ adopts non-zero values within the opening angle subtended by the emitting surface (not necessarily a sphere). We note that here and in the following we suppress the dependence of the intensity on the neutrino energy and assume that energy-integrated quantities are considered (the outlined formalism, however, is valid also for an energy-dependent treatment). The integration over $\omega$ at the observer's location can be substituted by an integration over the emitting surface of the source, because the radiation intensity is constant along rays, i.e.,

$I(D, \omega, t)=I_{\mathrm{o}}\left(\boldsymbol{R}_{\mathrm{o}}, \omega_{\mathrm{o}}, t\right)$

for any ray arriving at the observer from the source (and zero otherwise), where $\boldsymbol{R}_{\mathrm{O}}$ denotes the position of a surface element of the emitting surface in the coordinate frame of the source and $\omega_{0}$ the direction of the radiation field at that position toward the observer. Note that we ignore in Eq. (2) the trivial effect that the time $t$ for $I_{0}$ relative to the time for $I$ is subject to a retardation.

Moreover, in the following we disregard spectral and angular corrections that may be relevant when the emitting surface is in relative motion to the observer or sitting deep in the gravitational potential of a compact star (in which case general relativistic (GR) effects like redshifting and ray bending would be impor$\operatorname{tant}$ ). Considering the source to be at rest is a good assumption for the neutrinospheric region in the supernova core after bounce (the velocities of mass motions in this layer are unlikely to be higher than some $1000 \mathrm{~km} \mathrm{~s}^{-1}$, i.e., at most around one percent of the speed of light), while GR energy redshift is certainly of relevance on the $\sim 10-20 \%$ level during the proto-neutron star cooling phase ( $t \gtrsim 1 \mathrm{~s}$ after bounce), but much lower in the accretion and shock revival phases, when the forming neutron star is still considerably less compact than the final remnant.

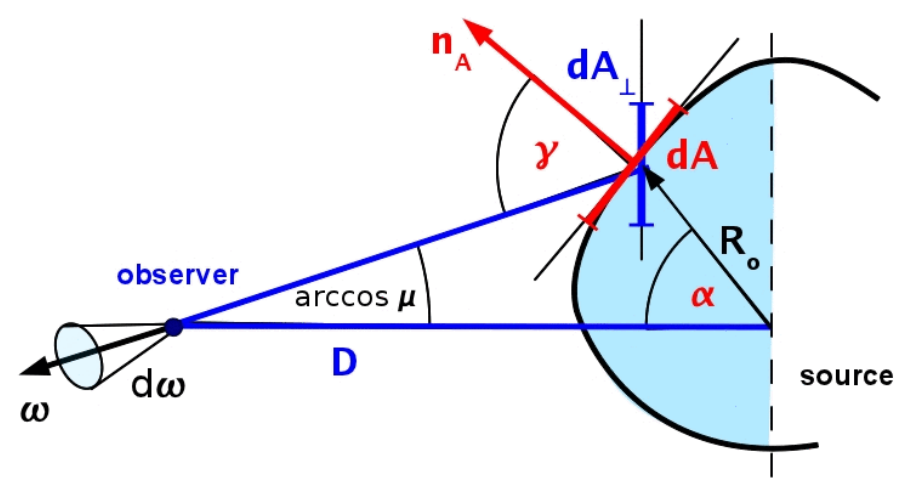

Fig. 1. Sketch illustrating the various quantities involved when deriving the observable luminosity of a radiating source, whose visible surface is shaded in blue. When the observer is at infinity, the emitting surface is a sphere, and $\boldsymbol{R}_{\mathrm{o}}$ is measured from the origin of this sphere, one obtains $\alpha=\gamma$.

For a distant observer $D \gg \max \left\{\left|\boldsymbol{R}_{\mathrm{o}}\right|\right\}$ holds, i.e., the value of $\mu$ is very close to one for the whole emitting surface. Denoting the solid angle subtended by a surface element of the emitting surface by $\mathrm{d} \omega$, we have $\mathrm{d} \omega=\mathrm{d} A_{\perp} / D^{2}$, where $\mathrm{d} A_{\perp}=\cos \gamma \mathrm{d} A$ is the projected area of this surface element perpendicular to the line of sight, when $\gamma$ is the angle between the normal unit vector $\boldsymbol{n}_{A}$ of the emitting surface element $\mathrm{d} A$ and the line of sight (see Fig. 1), but specified to the case $D \gg\left|\boldsymbol{R}_{\mathrm{o}}\right|$. Hence, we obtain for the observable luminosity the expression

$L_{\mathrm{o}}(t)=4 \pi D^{2} F(D, t)=4 \pi \int_{\text {vis.surf. }} \mathrm{d} A \cos \gamma I_{\mathrm{o}}\left(\boldsymbol{R}_{\mathrm{o}}, \omega_{\mathrm{o}}, t\right)$.

In order to evaluate the integral in Eq. (3), one needs to know the intensity $I_{\mathrm{o}}$ as a function of energy, emission direction, and time at every point of the radiating surface of the source. Determining $I_{\mathrm{o}}\left(\boldsymbol{R}_{\mathrm{o}}, \omega_{\mathrm{o}}, t\right)$ in general requires calculating full-scale neutrino transport. With this quantity as the solution of the transport problem at hand, Eq. (3) can be evaluated directly (in general with energy dependence) by performing the integration over any surface that encloses the radiation (neutrino) emitting source and that lies outside the volume where radiation interacts with matter (i.e., the intensity $I_{\mathrm{o}}$ in all points on the chosen surface must be given in the reference frame of the observer and must be preserved on the way from the emission point to the observer).

Our ray-by-ray transport approximation, however, yields only the local neutrino energy density $E\left(\boldsymbol{R}_{0}, t\right)$ and the neutrino flux density $F\left(\boldsymbol{R}_{\mathrm{o}}, t\right)$. To estimate the neutrinos radiated from every point of the neutrinosphere to the observer, we therefore have to develop an approach that yields a reasonable representation of the direction-dependent intensity as function of the quantities delivered by our transport approximation ${ }^{1}$. To this end, we assume that the neutrino distribution is axisymmetric around the normal vector $\boldsymbol{n}_{A}$ at all points $\boldsymbol{R}_{0}$. This implies that the direction dependence of the intensity $I_{0}$ is described by the direction angle $\gamma$ only (see Fig. 1), i.e., $I_{0}=I_{0}\left(\boldsymbol{R}_{0}, \gamma, t\right)$, and that the flux direction is given by $\boldsymbol{n}_{A}$. Assuming further that $I_{\mathrm{o}}(\gamma)$ can be approximated

\footnotetext{
1 Note that the procedure described in the following does not depend on whether the transport is performed in the gray approximation or is energy dependent. We therefore suppress the energy variable in all transport quantities and introduce mean energies in our gray treatment instead of considering neutrino energies as a function of spectral frequencies.
} 
by the lowest two terms of an expansion in spherical harmonics (as in the diffusion approximation), one can write

$I_{\mathrm{o}}\left(\boldsymbol{R}_{\mathrm{o}}, \gamma, t\right)=\frac{F_{\mathrm{o}}\left(\boldsymbol{R}_{\mathrm{o}}, t\right)}{2 \pi}\left(1+\frac{3}{2} \cos \gamma\right)$.

Because the radiation flux density is defined as the first angular moment of the intensity, one can easily verify that the numerical coefficient $3 / 2$ of the dipole term ensures that the flux density $F_{\mathrm{o}}\left(\boldsymbol{R}_{\mathrm{o}}, t\right)$ (normal to the emitting surface element $\mathrm{d} A$ ) is given by $\int_{0}^{2 \pi} \mathrm{d} \varphi \int_{0}^{1} \mathrm{~d} \cos \gamma \cos \gamma I_{\mathrm{o}}\left(\boldsymbol{R}_{\mathrm{o}}, \gamma, t\right)$, if the radiating surface does not receive any incident neutrinos from outside (i.e., $I_{\mathrm{o}}\left(\boldsymbol{R}_{\mathrm{o}}, \gamma, t\right)=0$ for $\left.\cos \gamma<0\right)^{2}$.

Inserting Eqs. (4) into (3), we find for the observable neutrino luminosity the expression

$L_{\mathrm{o}}(t)=2 \int_{\text {vis.surf. }} \mathrm{d} A \cos \gamma F_{\mathrm{o}}\left(\boldsymbol{R}_{\mathrm{o}}, t\right)\left(1+\frac{3}{2} \cos \gamma\right)$.

We further define an observable mean neutrino energy according to

$\langle E\rangle_{\mathrm{o}}(t)=\frac{L_{\mathrm{o}}(t)}{L_{\mathrm{n}, \mathrm{o}}(t)}$,

where

$L_{\mathrm{n}, \mathrm{o}}(t)=2 \int_{\text {vis.surf. }} \mathrm{d} A \cos \gamma \frac{F_{\mathrm{o}}\left(\boldsymbol{R}_{\mathrm{o}}, t\right)}{\epsilon\left(\boldsymbol{R}_{\mathrm{o}}, t\right)}\left(1+\frac{3}{2} \cos \gamma\right)$

is the observable neutrino number flux with $\epsilon$ being the mean energy of the neutrino energy spectrum radiated from point $\boldsymbol{R}_{\mathrm{o}}$.

Our $3 \mathrm{D}$ radiation hydrodynamics code computes the timedependent neutrino energy flux density, $F_{\mathrm{o}}\left(\boldsymbol{R}_{\mathrm{o}}, t\right)$, and neutrino number flux density, $F_{n, o}\left(\boldsymbol{R}_{\mathrm{o}}, t\right)$, through a sphere of radius $R_{\mathrm{O}}=$ $\left|\boldsymbol{R}_{\mathrm{o}}\right|$ in dependence of the angular position $\boldsymbol{\Omega} \equiv(\theta, \phi)$, but actually stores the related quantities

$\Lambda(\boldsymbol{\Omega}, t) \equiv 4 \pi R_{\mathrm{o}}^{2} F_{\mathrm{o}}\left(R_{\mathrm{o}}, \boldsymbol{\Omega}, t\right)$,

and

$\Lambda_{\mathrm{n}}(\boldsymbol{\Omega}, t) \equiv \frac{\Lambda(\boldsymbol{\Omega}, t)}{\epsilon(\boldsymbol{\Omega}, t)}=4 \pi R_{\mathrm{o}}^{2} F_{\mathrm{n}, \mathrm{o}}\left(R_{\mathrm{o}}, \boldsymbol{\Omega}, t\right)$,

because these quantities are constant in the ray-by-ray approximation of the free streaming region. In this approximation, both the neutrino energy flux and the neutrino number flux are purely radial.

\footnotetext{
${ }^{2}$ Note that the requirement $I_{0} \geq 0$ implies that Eq. (4) is valid in the whole range of $\cos \gamma \geq-\frac{2}{3}$, which includes inward going radiation for $\cos \gamma<0$. Extending the integration over all directions of validity, one obtains $\frac{25}{27} F_{\mathrm{o}}=\int_{0}^{2 \pi} \mathrm{d} \varphi \int_{-2 / 3}^{1} \mathrm{~d} \cos \gamma \cos \gamma I_{\mathrm{o}} \approx F_{\mathrm{o}}$ and $E_{\mathrm{o}}=\int_{0}^{2 \pi} \mathrm{d} \varphi \int_{-2 / 3}^{1} \mathrm{~d} \cos \gamma I_{\mathrm{o}}=\frac{35}{12} F_{\mathrm{o}} / c \approx 3 F_{\mathrm{o}} / c$. This means that we have $F_{\mathrm{o}} \approx \frac{1}{3} c E_{\mathrm{o}}$, which is a reasonably good approximation of the relation between flux density and energy density at the neutrinosphere, where one typically obtains $f_{\mathrm{o}} \equiv F_{\mathrm{o}} /\left(E_{\mathrm{o}} c\right) \approx \frac{1}{4} \ldots \frac{1}{3}$ for the flux factor at an optical depth between unity and about $2 / 3$ in detailed neutrino transport calculations in spherical symmetry (Janka 1991). The ansatz of Eq. (4) is therefore consistent with basic properties of the neutrinospheric emission. Moreover, we note that the expression corresponds to the limb-darkening law $I_{\mathrm{E}}(\cos \gamma) / I_{\mathrm{E}}(1)=(2 / 5)(1+3 / 2 \times \cos \gamma)$ that can be derived on grounds of the Eddington approximation (see, e.g., Mihalas 1978, p. 61; or Morse \& Feshbach 1953, p. 187).
}

Using Eqs. (8) and (9), and the fact that $\mathrm{d} A=R_{\mathrm{o}}^{2} \mathrm{~d} \Omega$ with $\mathrm{d} \Omega=\sin \theta \mathrm{d} \theta \mathrm{d} \phi$ for the special case of an emitting sphere of radius $R_{\mathrm{o}}$, we can rewrite the general expression for the observable neutrino luminosity given in Eq. (5) in the form

$L_{\mathrm{o}}(t)=\frac{1}{2 \pi} \int_{\text {vis.hem. }} \mathrm{d} \Omega \cos \gamma \Lambda(\boldsymbol{\Omega}, t)\left(1+\frac{3}{2} \cos \gamma\right)$

and that of the observable neutrino number flux given in Eq. (7) in the form

$L_{\mathrm{n}, \mathrm{o}}(t)=\frac{1}{2 \pi} \int_{\text {vis.hem. }} \mathrm{d} \Omega \cos \gamma \Lambda_{n}(\boldsymbol{\Omega}, t)\left(1+\frac{3}{2} \cos \gamma\right)$,

where in both cases the integration is performed over the visible hemisphere ${ }^{3}$.

For the evaluation of the gravitational wave amplitude in Sect. 4.1.2 we will also need the quantity

$\frac{\mathrm{d} \Lambda}{\mathrm{d} \Omega}(\boldsymbol{\Omega}, t) \equiv F_{\mathrm{o}}\left(R_{\mathrm{o}}, \boldsymbol{\Omega}, t\right) R_{\mathrm{o}}^{2}$

and the corresponding angle-integrated quantity

$\Lambda(t) \equiv \oint_{\text {surf. }} \mathrm{d} \Omega \frac{\mathrm{d} \Lambda}{\mathrm{d} \Omega}(\boldsymbol{\Omega}, t)=\frac{1}{4 \pi} \oint_{\text {surf. }} \mathrm{d} \Omega \Lambda(\boldsymbol{\Omega}, t)$.

For the later discussion of the results we finally define the surface-averaged neutrino flux density

$\left\langle F_{\mathrm{o}}\right\rangle(t) \equiv \frac{1}{4 \pi} \oint_{\text {surf. }} \mathrm{d} \Omega F_{\mathrm{o}}\left(R_{\mathrm{o}}, \boldsymbol{\Omega}, t\right) \equiv \frac{1}{4 \pi R_{\mathrm{o}}^{2}} \frac{\mathrm{d} \mathcal{E}(t)}{\mathrm{d} t}$,

where

$\frac{\mathrm{d} \mathcal{E}(t)}{\mathrm{d} t}=\oint_{\text {surf. }} \mathrm{d} \Omega \frac{\mathrm{d} \Lambda}{\mathrm{d} \Omega}(\boldsymbol{\Omega}, t)=\Lambda(t)$

is the total energy loss rate at time $t$ from the supernova core to all directions, which (because of the flux variations over the sphere) is no directly observable quantity.

We have also analyzed the evolution of the neutrino flux asymmetry by calculating the angular pseudo-power spectrum of the neutrino energy flux variation

$\delta_{\Lambda}(\boldsymbol{\Omega}, t) \equiv \frac{\Lambda(\boldsymbol{\Omega}, t)-\Lambda(t)}{\Lambda(t)}$,

where $\Lambda(\boldsymbol{\Omega}, t)$ and $\Lambda(t)$ are defined in Eqs. (8) and (13), respectively. The pseudo-power spectrum is given by the decomposition of $\delta_{\Lambda}(\boldsymbol{\Omega}, t)$ in spherical harmonic coefficients

$\Lambda_{l m}(t)=\oint \mathrm{d} \Omega \delta_{\Lambda}(\boldsymbol{\Omega}, t) Y_{l m}^{*}(\Omega)$,

where $Y_{l m}^{*}(\Omega)$ is the respective (complex conjugate) spherical harmonics. For our mode analysis we actually used the pseudopower coefficients $C_{0} \equiv\left|\Lambda_{00}\right|^{2}$ and

$C_{l} \equiv \frac{1}{2 l+1}\left(\left|\Lambda_{l 0}\right|^{2}+2 \sum_{m=1}^{m=l}\left|\Lambda_{l m}\right|^{2}\right)$

for $l>0$, respectively.

${ }^{3}$ Using of the ray-by-ray approximation has the advantage that the evaluation of the integrals on the rhs of Eqs. (5) and (7) does not require the specification of a suitable surface, but can be done on any sphere outside the neutrino-decoupling region, as Eqs. (10) and (11) are independent of $\boldsymbol{R}_{\mathrm{o}}$. 


\subsection{Results}

The evolution of our models can be divided into four distinct phases (Figs. 2, 3).

(1) The first phase, the quasi-spherical shock-expansion phase (Fig. 2, top row), lasts from shock formation shortly after core bounce to $80-150 \mathrm{~ms}$, when convection sets in. During this phase the shock rapidly propagates out to a radius of $\sim 200 \mathrm{~km}$, where its expansion comes to a halt.

(2) The second phase, the hydrodynamically vigorous preexplosion phase, comprises the growth of post-shock convection and of the standing accretion shock instability, SASI (Fig. 2, second row from top).

(3) The post-explosion accretion phase begins when energy deposition by $v$-heating in the post-shock layers becomes sufficiently strong to launch the explosion, and the total energy in the post-shock region ultimately becomes positive (see Sect. 2.3 for a definition). During this phase the shock accelerates outward while gas is still accreted onto the PNS. This process is commonly called "shock revival" (Fig. 2, third row from top).

Non-radial instabilities during the latter two stages cause considerable temporal and angular fluctuations of the neutrino energy flux density as illustrated in Figs. 3-5. Besides the evolution of the shock radius, the figures show the surface-averaged neutrino light curve $\Lambda(t)$, i.e., the total energy loss due to neutrinos versus time (Eqs. (13), (15)), together with the time evolution of the maximum and minimum values of $\Lambda(\Omega, t)$ (Eq. (8); the numerical evaluation is performed on an arbitrarily chosen sphere of $500 \mathrm{~km}$ radius). Distinct and high-amplitude spikes in $\Lambda_{\max }(\Omega, t)$ are visible for several $100 \mathrm{~ms}$ and reflect violent post-shock convection, possible SASI activity, and anisotropic accretion fluctuations after the onset of the explosion. We have marked the explosion time $t_{\exp }$ (see Sect. 2.3, and Table 1) by a vertical dashed line in Figs. 3 and 4. The post-explosion accretion phase lasts until $\sim 500 \mathrm{~ms}$ (models W15-4 and N20-2) or $\sim 700 \mathrm{~ms}$ (model L15-3) depending on the progenitor.

(4) During the post-accretion phase, the fourth and final phase characterizing the evolution of our models (Fig. 2, bottom row), gas infall to the proto-neutron star has come to an end and the newly formed neutron star looses mass at a low rate in a nearly spherical neutrino-driven wind. We find considerably less temporal variability and a lower level of angular variation $(\$ 10 \%)$ of the neutrino emission during this fourth phase (Figs. 3-5).

While in model L15-3 the amplitudes of the neutrino emission fluctuations decrease continuously, the other two models exhibit growing temporal emission variations (though at a lower level than the earlier variability) during a later stage (notice the decrease/increase in $\Lambda_{\max }-\Lambda_{\min }$ in Figs. 3 and 4), which might be considered as a fifth evolutionary phase. This phase is associated with growing convective activity below the neutrinosphere. This PNS convection develops more or less strongly in the different models depending on the location of the convectively unstable region relative to the inner radial boundary of our computational domain.

We have evaluated the time evolution of the neutrino energy flux asymmetry by producing $4 \pi$-maps that show the relative angular variation $\Delta F_{\mathrm{o}} /\left\langle F_{\mathrm{o}}\right\rangle$ of the total (i.e., sum of all neutrino flavors) neutrino energy flux density across a sphere (normalized to the surface-averaged flux density; Eq. (14)). Several snapshots of this evolution are shown for model W15-4 in Fig. 5.
The evolution of the typical angular scales of the fluctuations is reflected by the pseudo-power spectrogram of the electron neutrino energy flux variation (Eq. (16)) in Fig. 6, top panels, which give the color-coded pseudo-power coefficient distribution normalized to the maximum value versus time. The variation of the pseudo-power coefficients with angular mode number is shown in Fig. 7 at selected times of $200 \mathrm{~ms}$ (blue), $400 \mathrm{~ms}$ (red), and $1000 \mathrm{~ms}$ (black).

During the quasi-spherical shock expansion phase the level of angular fluctuations of $F$ is low $\left(\lesssim 10^{-2}\right)$, while the fluctuation amplitudes of the total neutrino energy flux density reach a level of several $10 \%$ during the hydrodynamically vigorous second phase and the post-explosion accretion phase, where a few distinct regions or even single spots with an angular size of $10^{\circ}$ to $20^{\circ}$ dominate the emission (Fig. 5, panels 2 and 3). The mode number $l$ of the dominant angular perturbation scale is of no relevance during the first phase, as the maximum pseudo-power coefficient $C_{l}^{\max }$ (see Eq. (18)) is tiny $\lesssim 10^{-6}$ (Fig. 6, middle panels), i.e., the dominating $l=2$ and $l=4$ modes visible in the upper panels of Fig. 6 only reflect tiny angular perturbations imprinted presumably by the computational grid. When neutrino heating eventually causes significant non-radial flow during the second and third phases, $C_{l}^{\max }$ rises sharply to a level of $\sim 10^{-3}$ (Fig. 6 , middle panels), and the relative angular variations of the electron neutrino flux density grow to the several ten percent level (Fig. 6, bottom panels). The latter quantity gives the maximum minus the minimum flux density on the sphere divided by the angle-averaged flux density in percent. Compared to the total neutrino emission in Figs. 3-5, the temporal and angular variations in different directions are even more pronounced when considering the energy flux of the electron neutrinos or electron anti-neutrinos alone (Fig. 6), where angular variations can exceed $100 \%$ in all models during the pre-explosion and accretion phases, and peak values are close to $200 \%$ during short episodes (Fig. 6, lower panels).

During the vigorous pre-explosion phase including the postexplosion accretion stage, electron neutrinos and antineutrinos dominate the angular flux variations, while muon and tau neutrinos (accounting for roughly 50\% of the total luminosity) exhibit essentially isotropic emission in all directions. The reason of this finding is that $v_{\mathrm{e}}$ and $\bar{v}_{\mathrm{e}}$ are produced almost exclusively by efficient charged-current reactions in the accretion region perturbed by non-radial fluid flows. The spectrogram of the two phases is characterized by initially very small-scale angular variations with $l \gtrsim 12$, which are associated with the onset of the Rayleigh-Taylor overturn activity, and which merge to continuously larger angular structures that correspond to $l \approx$ $1 \ldots 4$ modes toward the end of the accretion period at $0.4-0.6 \mathrm{~s}$ (depending on the model). This evolution is accompanied by a steady decrease of $C_{l}^{\max }$ to a level of $\sim 10^{-5}$ and a reduction of the electron neutrino flux density variations from values well beyond $100 \%$ to a level of $\sim 10 \%$, only (see Fig. 6, left panels).

When neutrino-energy deposition in the post-shock layers becomes sufficiently strong and the explosion is eventually launched at about 250 to $500 \mathrm{~ms}$ (depending on the model; Table 1), subsequent radial shock expansion rapidly diminishes the activity of the SASI and freezes post-shock convection. Single, longer lasting downdrafts of accretion flows are associated with isolated hot spots, where the variations of the total flux density can reach peak amplitudes of up to $\sim 70 \%$ (Fig. 5, panel 3). When accretion has ended, the amplitude of the angular variations of the total neutrino energy flux reduces to a level of a few percent (Figs. 3, 4), and the angular pattern of the emission 
E. Müller et al.: Parametrized 3D models of neutrino-driven supernova explosions
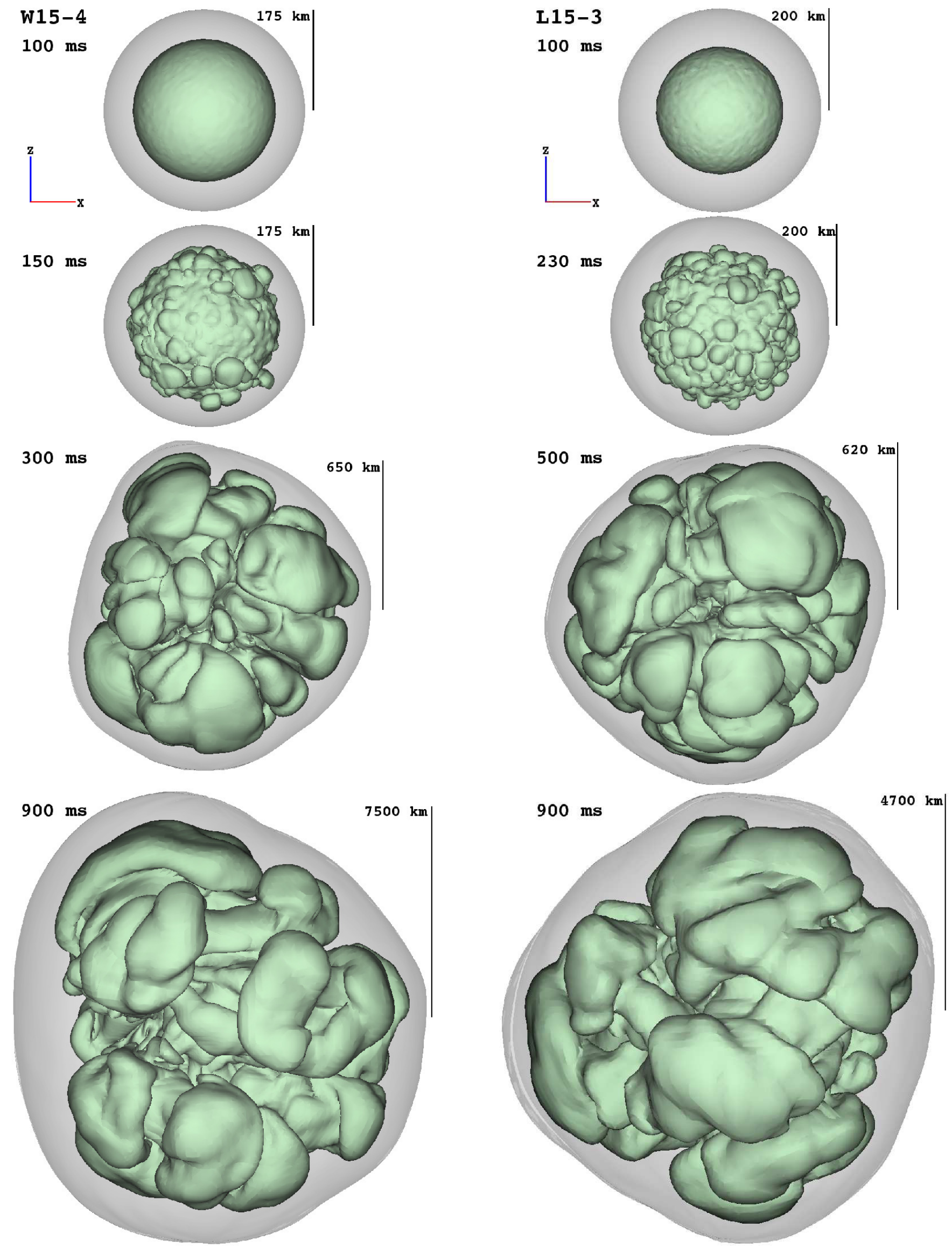

Fig. 2. Snapshots of models W15-4 (left) and L15-3 (right) illustrating the four phases characterizing the evolution of our 3D models (see text for details). Each snapshot shows two surfaces of constant entropy marking the position of the shock wave (gray) and depicting the growth of non-radial structures (greenish). The time and linear scale are indicated for each snapshot. 

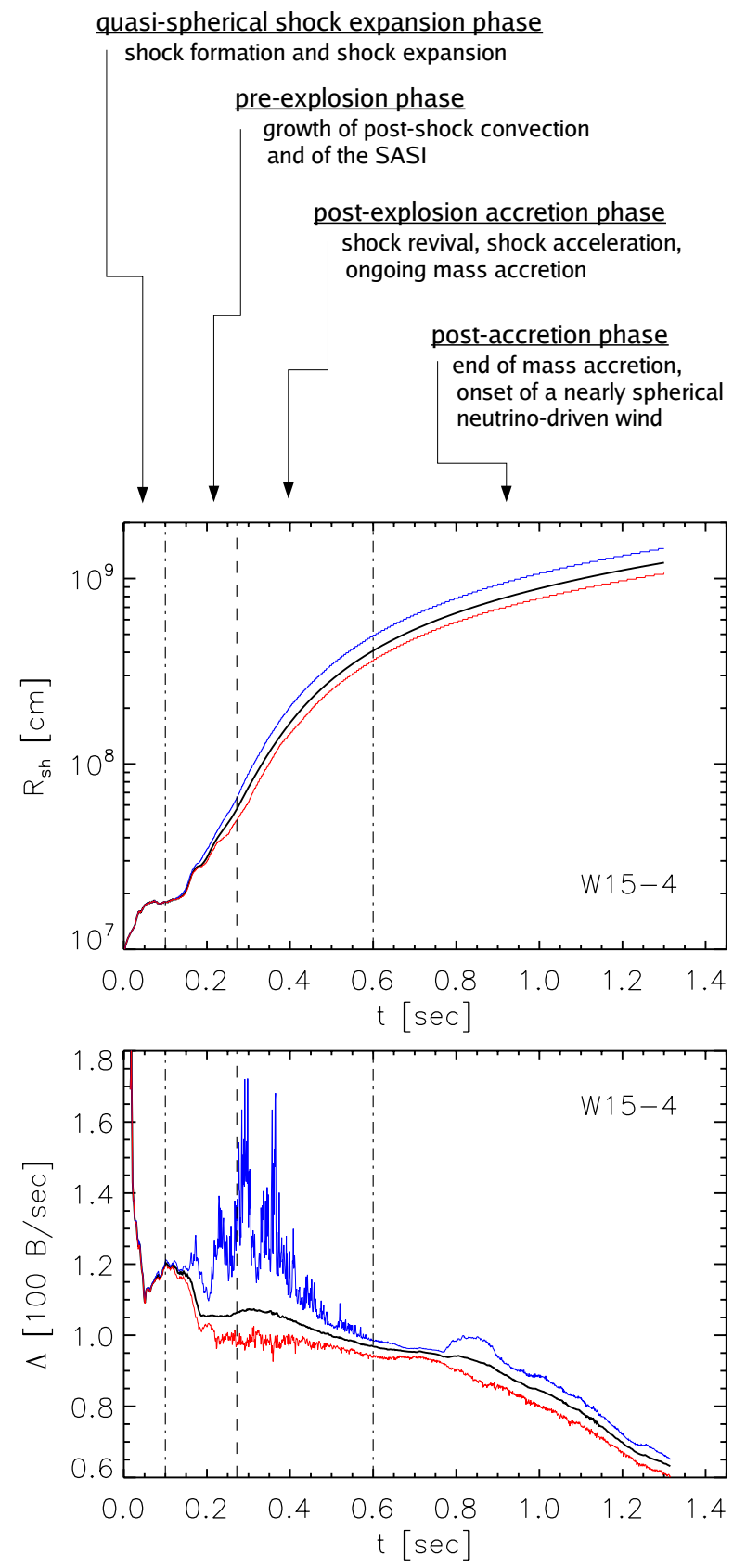

Fig. 3. Shock radius (top) and total (i.e., summed over all flavors) energy loss rate due to neutrinos (bottom) as functions of time for model W15-4. In the upper panel, the black curve shows the angleaveraged mean shock radius, the blue (red) curve gives the maximum (minimum) shock radius, and the vertical dashed line marks the time of the onset of the explosion as defined in Sect. 2.3. In the lower panel, the blue and red curves show the time evolution of $\Lambda_{\max }(\Omega, t)$ and $\Lambda_{\min }(\Omega, t)$, the maximum and minimum value of $\Lambda(\Omega, t)$ (Eq. (8)) on a sphere of $500 \mathrm{~km}$ radius, respectively. The black line gives the corresponding surface-averaged value $\Lambda(t)$ (Eq. (13)). Note that the luminosities imposed at the inner radial grid boundary are kept constant during the first second and later are assumed to decay like $t^{-2 / 3}$.

becomes more uniform over the sphere, consisting of many spots with an angular size of $\sim 30^{\circ}$ (Fig. 5, panel 4).

In the early post-accretion phase of model W15-4, $0.6 \mathrm{~s} \lesssim$ $t \lesssim 0.8 \mathrm{~s}$, the spectrogram indicates the presence of lowamplitude $\left(C_{l}^{\max } \lesssim 10^{-4}\right)$, small-angular size $(l \gtrsim 10)$ perturbations in the electron neutrino energy flux caused by some low-amplitude turbulent flow in and below the neutrinospheric
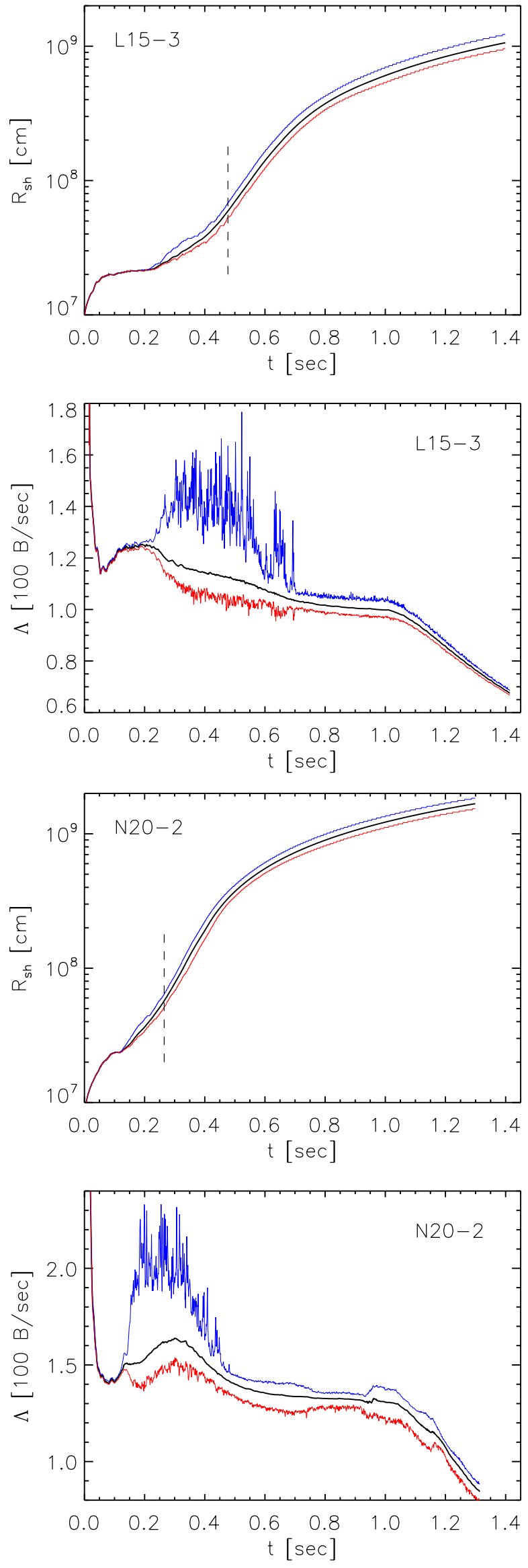

Fig. 4. Same as Fig. 3 but for models L15-3 (uppermost two panels) and N20-2 (lowermost two panels), respectively. 


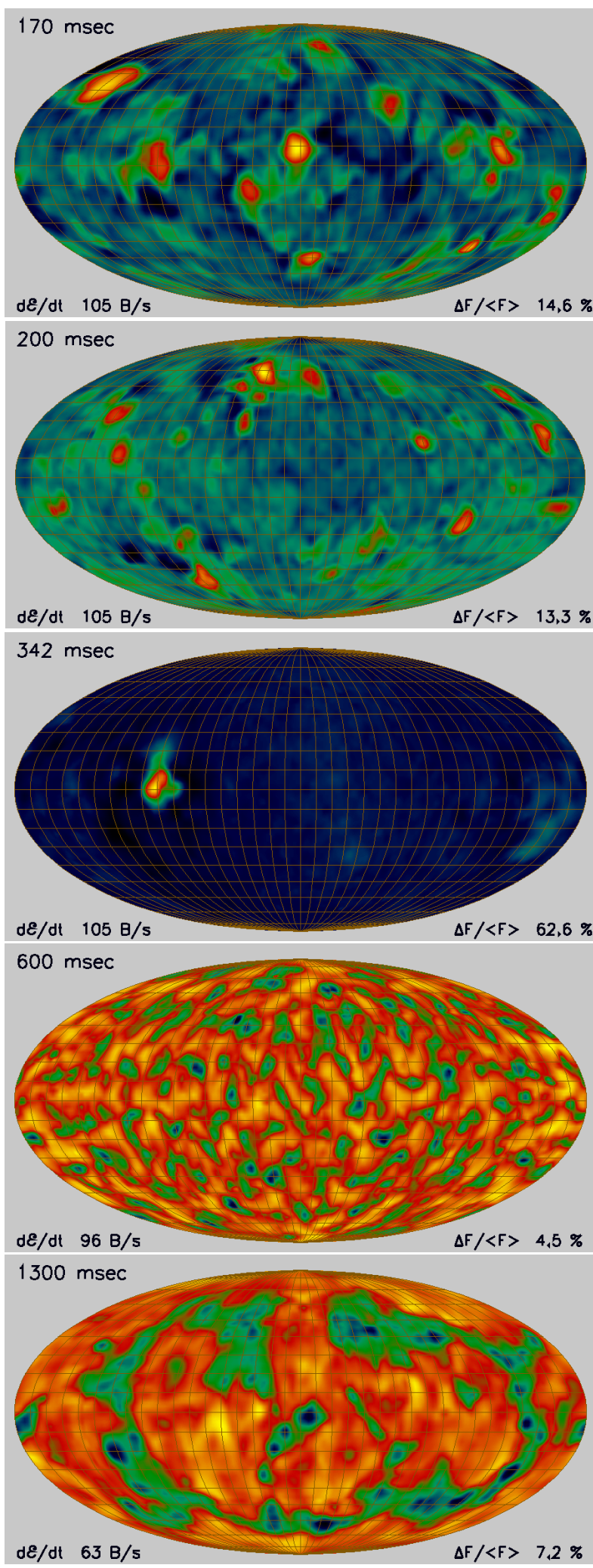

Fig. 5. Neutrino flux asymmetry at $170 \mathrm{~ms}, 200 \mathrm{~ms}, 342 \mathrm{~ms}, 600 \mathrm{~ms}$, and $1.3 \mathrm{~s}$ (from top to bottom), respectively. The $4 \pi$-maps show the relative angular variation $\Delta F_{\mathrm{o}} /\left\langle F_{\mathrm{o}}\right\rangle$ of the total (i.e., sum of all neutrino flavors) neutrino energy flux density over a sphere (normalized to its angular average) for model W15-4. The maximum value is given in the lower right corner of each panel. Regions of higher emission are shown in bright yellow, while orange, red, green, and blue colors indicate successively less emission. Note that the color scale of each panel is adjusted to the maximum and minimum values at the corresponding time. The total energy loss rate due to neutrinos is given in the lower left corner. region. When strong convection inside the PNS is encountered for $t \gtrsim 0.8 \mathrm{~s}$ the spectrogram drastically changes, being dominated by angular modes with $l=4$, but still with $C_{l}^{\max } \sim 10^{-4}$. The electron neutrino flux density variations rise somewhat to a level of $10 \%$ to $20 \%$, and become manifest in the total energy loss rate, too (Figs. 3, 4).

Model N20-2 exhibits quite a similar behavior as model W15-4 except for the appearance of even larger $(l \sim 3)$ angular structures clearly recognizable in the pseudo-power spectrogram between $1.0 \mathrm{~s}$ and $1.2 \mathrm{~s}$ (Figs. 6,7). This differs from the behavior of model L15-3, where the amplitudes and angular size of the energy flux density variations remain small and even decrease in the post-explosion phase (Figs. 4, 6).

The reason for the fluctuation behavior of the neutrino emission during the vigorous pre-explosion and post-explosion accretion phases has been discussed, but what causes the spatial and temporal variations during the post-accretion phase? Because the explosion is well on its way at this time, neither post-shock convection nor the SASI nor accretion can be responsible. Hence, there only remains non-radial gas flow in the outer layers of the proto-neutron star. Ledoux convection in the proto-neutron star thus may become visible eventually, i.e., its presence in the inner parts of the computational domain may become dominant in observable signals. This happens in models W15-4 and N20-2, where the level of the non-radial specific kinetic energy $\left(v_{\theta}^{2}+v_{\phi}^{2}\right) / 2$, volume-averaged over the computational domain, inside the neutrinosphere shows a steep rise at $\sim 0.8 \mathrm{~s}$ and $\sim 0.9 \mathrm{~s}$, respectively (Fig. 8 ). These non-radial flows that develop in models W15-4 and N20-2 at late times also become manifest in all discussed quantities: $\Lambda_{\max }(\Omega, t), \Lambda_{\min }(\Omega, t)$, $C_{l}^{\max }$, the dominant low $l$-modes $(2 \lesssim l \lesssim 4)$, and relative angular flux-density variations. In contrast, no such effect is present in model L15-3 (see Fig. 8), where we find a steady decrease of $\Lambda_{\max }(\Omega, t)-\Lambda_{\min }(\Omega, t)$, higher $l$-modes $(l \gtrsim 10)$, smaller $C_{l}^{\max }$, and lower flux-density variation amplitudes than in models W15-4 and N20-2 (see Figs. 3, 4, and 6).

Simulations with fully self-consistent treatment of the PNS interior show the presence of convection inside the PNS, i.e., below the neutrinosphere (see Keil et al 1996; Buras et al. 2006; Dessart et al. 2006) more or less from the early post-bounce phase on. With the use of our inner radial grid boundary excising the inner parts of the PNS, and imposing neutrino luminosities at this boundary, convective activity is triggered only when the neutrino energy (or lepton number) inflow into the layers close to the grid boundary is faster than neutrino transport can carry away this energy (or lepton number). Then convectively unstable gradients develop and convective flows begin to carry the energy and lepton-number outward. Whether this happens or not depends on the boundary luminosities as well as on the location of the grid boundary within the density and temperature profiles of the PNS layers below the neutrinosphere. That location determines the efficiency of the neutrino transport and varies with the stellar progenitor, whose mass-infall rate decides how much mass accumulates in the near-surface layers of the PNS outside the inner grid boundary. The relative strength of the artificially imposed inflow of neutrino energy and lepton number compared to the efficiency of the neutrino transport on the grid, both sensitive to the location and contraction of the grid boundary on the one hand and the chosen values of the boundary luminosities on the other, therefore decides about when, where, and how strongly convective activity develops below the neutrinosphere.

Because the position of and the conditions imposed at the inner boundary can thus influence the neutrino emission properties, in particular during the post-accretion phase, our respective 


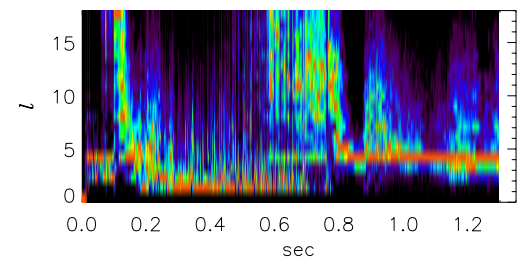

W15-4
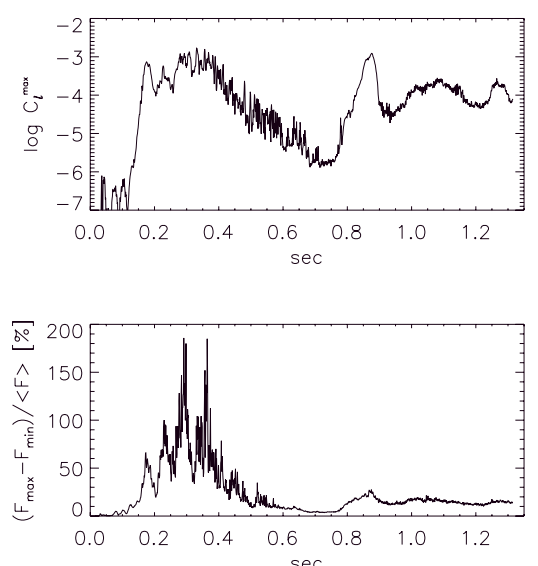

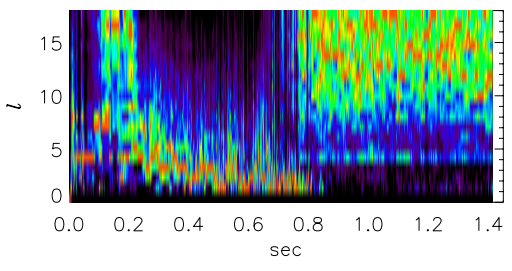

L15-3
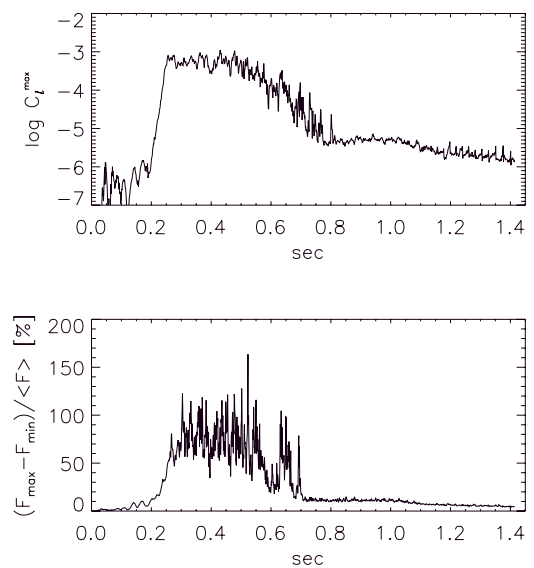

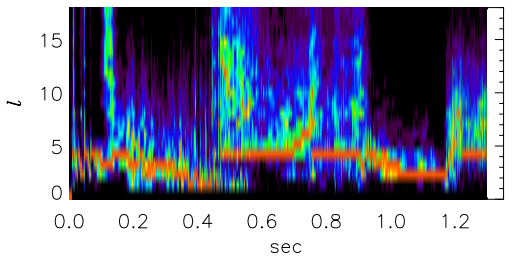

$\mathrm{N} 20-2$
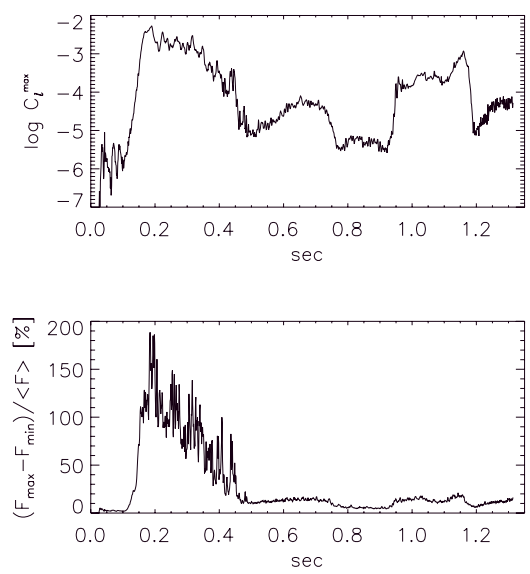

Fig. 6. Pseudo-power spectrogram of the electron-neutrino energy flux density (top row) for models W15-4 (left), L15-3 (middle), and N20-2 (right), respectively. The panels in the middle row show the corresponding maximum pseudo-power coefficient $C_{l}^{\max }$ as a function of time, and the panels in the lower row give the relative angular variation of the electron neutrino flux density (maximum minus minimum flux density on the sphere divided by the angle-averaged flux density in percent) with time.

model predictions must be considered with care. While they do not allow us to make any definite statements concerning the neutrino signal of a particular progenitor model because of the neglected treatment of the inner parts of the proto-neutron star, the models nevertheless show that convective flows below the neutrinosphere are likely to imprint themselves on the neutrino emission, and hence also on the GW signal of core-collapse supernovae. A measurement of these signals may actually provide some insight into the conditions inside proto-neutron stars.

Because the neutrino energy flux density varies in our models both with latitude and longitude, the observable neutrino luminosity $L_{\mathrm{o}}(t)$ is obtained by an integral over the hemisphere visible to an observer (Eq. (10)). In Fig. 9 we show the observable electron neutrino and electron anti-neutrino luminosities for one chosen viewing direction for the three models W15-4, L15-3, and N20-2, respectively. The results for other directions look very similar with all characteristic features being independent of the observer position. We provide these quantities in addition to the total neutrino energy loss rate (Eqs. (13) and (15); Figs. 3 and 4), because their temporal evolutions are the ones expected to be measurable in the IceCube and Super-Kamiokande detectors. These detectors (mainly for $\bar{v}_{\mathrm{e}}$ ) will be sensitive to a combination of the observable neutrino luminosity $L_{\mathrm{o}}$ and the observable mean neutrino energy $\langle E\rangle_{0}$. Thus, we also provide in Fig. 9 the time evolution of the observable mean neutrino energy and of the combination $L_{\mathrm{o}}\langle E\rangle_{\mathrm{o}}^{2}$, which (roughly) enters the IceCube detection rate of Cherenkov photons originating from the dominant inverse beta decay reaction $\bar{v}_{\mathrm{e}}+p \rightarrow n+e^{+}$ (Lund et al. 2010) ${ }^{4}$. Again one can recognize the different evolution stages, and in particular the post-shock convection and SASI phase, during which the quantity $L_{0}\langle E\rangle_{\mathrm{o}}^{2}$ exhibits rapid low-amplitude variations for all three models. The level of the

\footnotetext{
${ }_{4}$ Note that our transport approximation only provides luminosities and mean energies, but not the higher moments of the energy spectrum (see Sect. 3.1).
}

variations is a few percent (Fig. 9), which is considerably lower than that of the angular fluctuation amplitudes of the flux density, which reaches almost $100 \%$ for the total neutrino flux density (Fig. 5) and almost 200\% for the electron neutrino and electron antineutrino flux densities (Fig. 6, lower panels). However, because the flux density variations are caused by a few individual hot spots covering only angular areas of size $\sim(\pi / 9)^{2}$, the observable fluctuations (of $L_{\mathrm{o}}$ and $\langle E\rangle_{\mathrm{o}}$ ) are lower by a factor of roughly $(\pi / 9)^{2} /(2 \pi) \sim 1 / 50$. Some of this activity is also present at late times in the two models W15-4 and N20-2, where Ledoux convection develops in the simulated outer parts of the protoneutron star (see discussion above).

From the results presented above we conclude that the signals carry clear information about the postshock hydrodynamic activity, and about the duration and decay of the accretion period. Composition-shell interfaces present in the progenitor star can also leave an imprint. In model W15-4 the transition from the Fe-core to the $\mathrm{Si}$-shell manifests itself in fast drops of the luminosities of $v_{\mathrm{e}}$ and $\bar{v}_{\mathrm{e}}$ at $\sim 150 \mathrm{~ms}$, when the mass accretion rate decreases steeply at the time the interface between the Fe-core and the $\mathrm{Si}$-shell of the $15 M_{\odot}$ progenitor falls through the shock.

\section{Gravitational wave signature}

Non-radial mass motions caused by gravity waves in the nearsurface layers of the PNS, which are caused by the SASI and convection in the post-shock region as well as by convective activity inside the proto-neutron star (Murphy et al. 2009; Marek et al. 2009) (driven by Ledoux unstable lepton or entropy gradients) result in a time-dependent, aspherical density stratification that produces gravitational radiation. The anisotropic emission of neutrinos associated with the non-radial mass flow (see Sect. 3) contributes to the gravitational wave signal, too. We computed and analyzed the signature of this gravitational radiation for the 3D models discussed in Sect. 2.3. 
E. Müller et al.: Parametrized 3D models of neutrino-driven supernova explosions
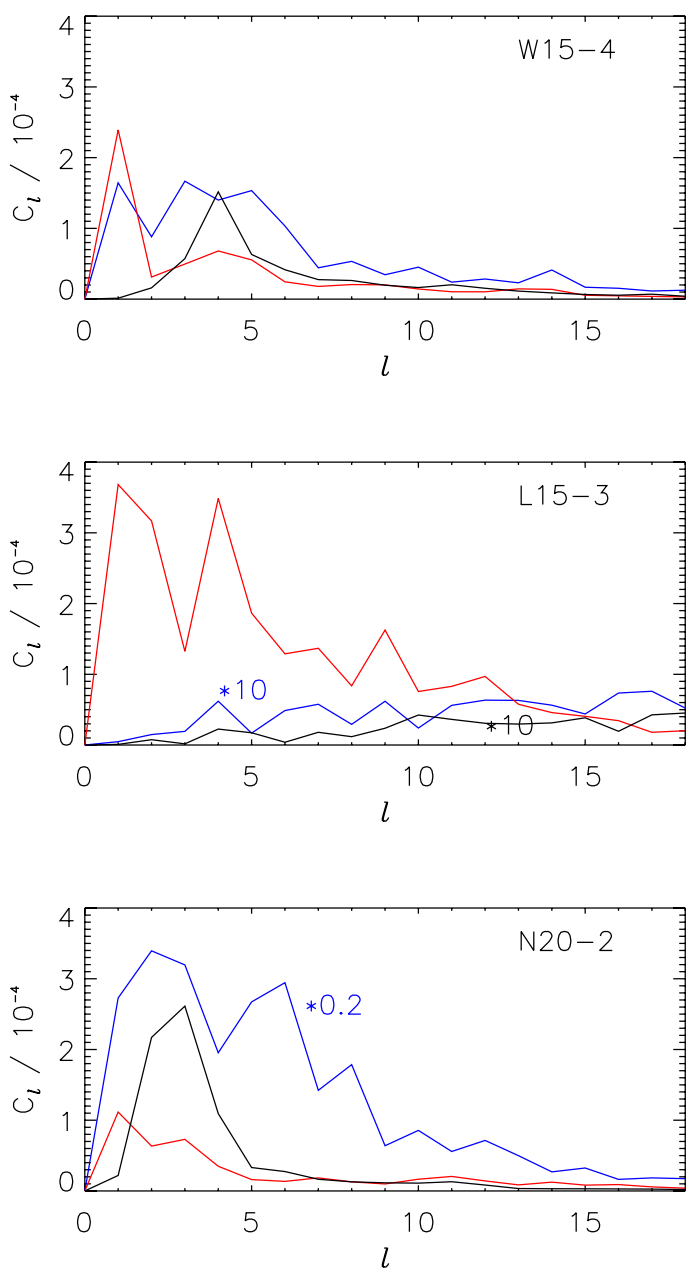

Fig. 7. Pseudo-power coefficients $C_{l}^{\max }$ of the electron-neutrino flux density as functions of angular mode number $l$ at $200 \mathrm{~ms}$ (blue), $400 \mathrm{~ms}$ (red), and 1000 ms (black) for models W15-4 (top), L15-3 (middle), and N20-3 (bottom), respectively.

\subsection{Formalism}

\subsubsection{Non-radial mass flow}

If a source is of genuine three-dimensional nature, as it is the case for our models, it is common to express the gravitational quadrupole radiation tensor, $\boldsymbol{h}^{\mathrm{TT}}$, in the transverse traceless gauge in the following tensorial form

$\boldsymbol{h}^{\mathrm{TT}}(\boldsymbol{X}, t)=\frac{1}{R}\left(A_{+} \boldsymbol{e}_{+}+A_{\times} \boldsymbol{e}_{\times}\right)$

(see, e.g., Misner et al. 1993). $R$ denotes the distance between the observer and the source, and the unit linear-polarization tensors are given by

$\boldsymbol{e}_{+}=\boldsymbol{e}_{\theta} \otimes \boldsymbol{e}_{\theta}-\boldsymbol{e}_{\phi} \otimes \boldsymbol{e}_{\phi}$,

$\boldsymbol{e}_{\times}=\boldsymbol{e}_{\theta} \otimes \boldsymbol{e}_{\phi}+\boldsymbol{e}_{\phi} \otimes \boldsymbol{e}_{\theta}$,

with $\boldsymbol{e}_{\theta}$ and $\boldsymbol{e}_{\phi}$ being the unit polarization vectors in $\theta$ and $\phi$-direction of a spherical coordinate system, and $\otimes$ denoting the tensor product.

The wave amplitudes $A_{+}$and $A_{\times}$represent the only two independent modes of polarization in the TT gauge (Misner et al. 1993). In the slow-motion limit, they are obtained from linear combinations of the second time derivatives (evaluated at retarded time, and denoted by a double dot accent) of the

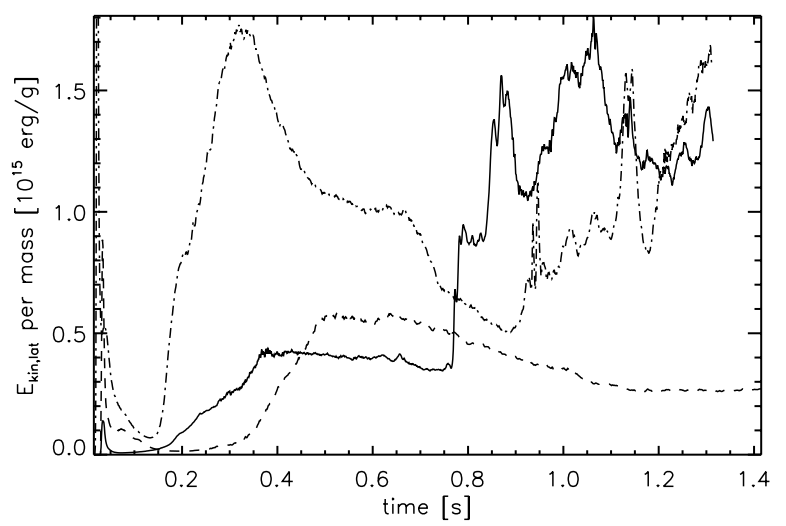

Fig. 8. Evolution of the non-radial specific kinetic energy $\left(v_{\theta}^{2}+v_{\phi}^{2}\right) / 2$ volume averaged over the computational domain inside the neutrinosphere for models W15-4 (solid), L15-3 (dashed), and N20-2 (dashed-dotted), respectively.
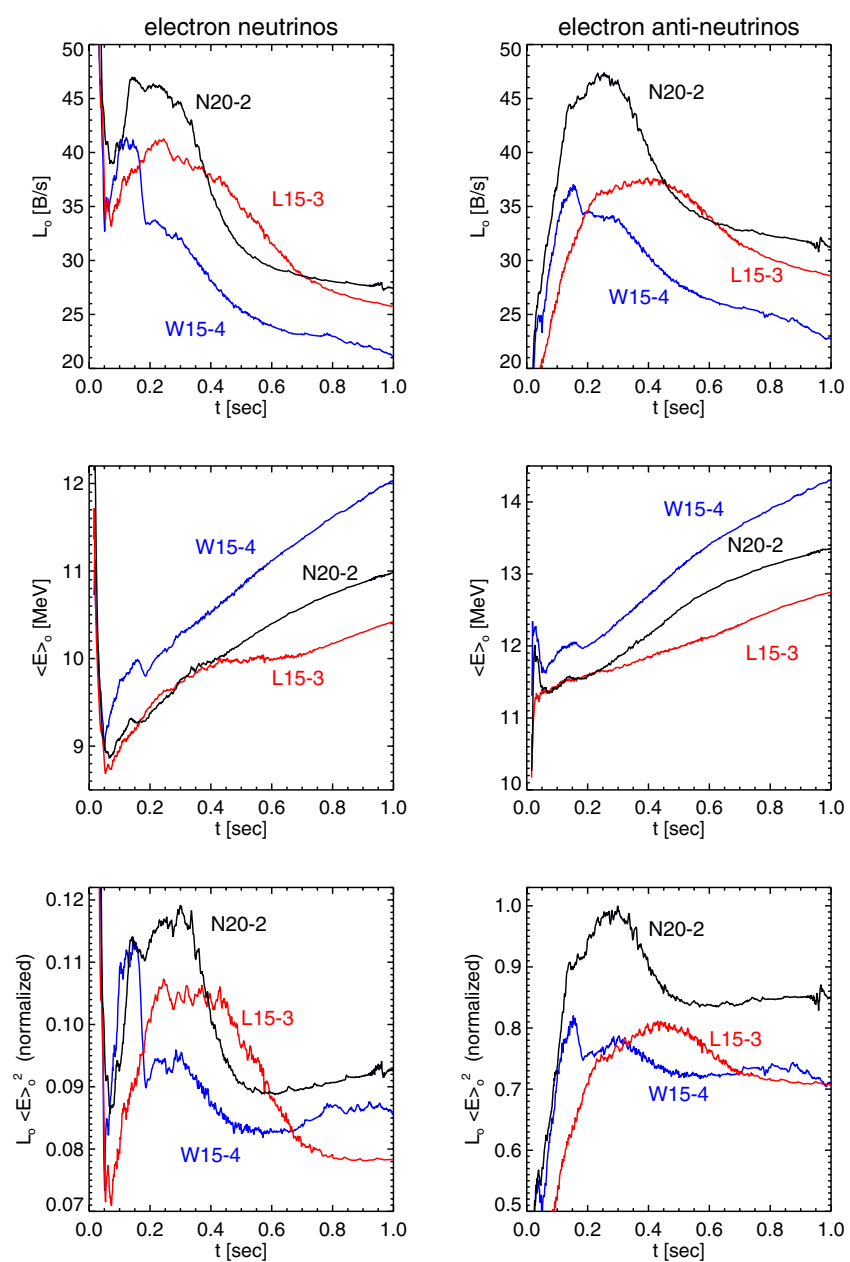

Fig. 9. Observable luminosity $L_{\mathrm{o}}$ (top row), observable mean energy $\langle E\rangle_{\mathrm{o}}$ (middle row), and normalized quantity $L_{\mathrm{o}}\langle E\rangle_{\mathrm{o}}^{2}$ (bottom row) of electron neutrinos (left column) and electron anti-neutrinos (right column) as a function of time for three of our models. Although we only present the results for one particular observer direction here, the global behavior and characteristics are very similar for all viewing directions.

components of the transverse traceless mass quadrupole tensor (Misner et al. 1993)

$A_{+}=\ddot{Q}_{\theta \theta}-\ddot{Q}_{\phi \phi}$,

$A_{\times}=2 \ddot{Q}_{\theta \phi}$. 
We computed the latter using a post-Newtonian approach whereby the numerically troublesome second-order time derivatives of the mass quadrupole tensor components are transformed into much better tractable spatial derivatives. Following Nakamura \& Oohara (1989) and Blanchet et al. (1990), the second-order time derivatives read in a Cartesian orthonormal basis (the spatial indices $i$ and $j$ run from 1 to 3 )

$\ddot{Q}_{i j}=\frac{G}{c^{4}} \int \mathrm{d}^{3} x \rho\left(2 v_{i} v_{j}-x_{i} \partial_{j} \Phi_{\mathrm{eff}}-x_{j} \partial_{i} \Phi_{\mathrm{eff}}\right)$,

where $G$ is Newton's gravitational constant, $c$ the speed of light in vacuum, $\Phi_{\text {eff }}$ the effective Newtonian gravitational potential including the general relativistic "case A" correction of the monopole term according to Marek et al. (2006), $\rho$ the massdensity, $v_{i}$ the Cartesian velocity components, and $\partial_{i}$ the partial derivative with respect to the coordinate $x^{i}$ of a Cartesian basis.

We note that the integrand in Eq. (24) has compact support and is known to the (2nd order) accuracy level of the numerical scheme employed in the hydrodynamics code. It can easily be shown that evaluating the integral of Eq. (24) by an integration scheme (of at least 2 nd order) is by one order of accuracy superior to twice applying numerical time-differentiation methods to quadrupole data given at discrete points of time (Finn \& Evans 1990; Mönchmeyer et al. 1991).

Exploiting the coordinate transformation between the orthonormal Cartesian basis $x^{i}$ and the orthonormal basis in spherical coordinates $\hat{x}^{i}$ (with $\hat{x}^{i} \in[r, \theta, \phi]$ ), the wave amplitudes $A_{+}$ and $A_{\times}$(Eqs. (22) and (23)) are obtained from the following second time derivatives of the spherical components of the mass quadrupole tensor (Oohara et al. 1997; Scheidegger et al. 2008)

$$
\begin{aligned}
I_{\theta \theta}^{\mathrm{TT}}= & \left(I_{x x}^{\mathrm{TT}} \cos ^{2} \phi+I_{y y}^{\mathrm{TT}} \sin ^{2} \phi+2 I_{z z}^{\mathrm{TT}} \sin \phi \cos \phi\right) \cos ^{2} \theta \\
& +I_{y y}^{\mathrm{TT}} \sin ^{2} \theta-2\left(I_{x z}^{\mathrm{TT}} \cos \phi+I_{y z}^{\mathrm{TT}} \sin \phi\right) \sin \theta \cos \theta, \\
I_{\phi \phi}^{\mathrm{TT}}= & I_{x x}^{\mathrm{TT}} \sin ^{2} \phi+I_{y y}^{\mathrm{TT}} \cos ^{2} \phi-2 I_{x y}^{\mathrm{TT}} \sin \phi \cos \phi, \\
I_{\theta \phi}^{\mathrm{TT}}= & \left(I_{y y}^{\mathrm{TT}}-I_{x x}^{\mathrm{TT}}\right) \cos \theta \sin \phi \cos \phi+I_{x y}^{\mathrm{TT}} \cos \theta\left(\cos ^{2} \phi\right. \\
& \left.-\sin ^{2} \phi\right)+I_{x z}^{\mathrm{TT}} \sin \theta \sin \phi-I_{y z}^{\mathrm{TT}} \sin \theta \cos \phi,
\end{aligned}
$$

where we used the abbreviation

$$
I_{i j}^{\mathrm{TT}} \equiv \ddot{Q}_{i j}^{\mathrm{TT}} \text {. }
$$

Choosing $\phi=0$ one obtains the polarization modes (see, e.g., Misner et al. 1993)

$$
\begin{aligned}
& A_{+}=I_{x x}^{\mathrm{TT}}-I_{y y}^{\mathrm{TT}}, \\
& A_{\times}=2 I_{x y}^{\mathrm{TT}}
\end{aligned}
$$

for $\theta=0$, and

$A_{+}=I_{z z}^{\mathrm{TT}}-I_{y y}^{\mathrm{TT}}$,

$A_{\times}=-2 I_{y z}^{\mathrm{TT}}$,

for $\theta=\pi / 2$, respectively. These expressions were already discussed in earlier investigations concerned with the evaluation of the gravitational wave signature of 3D core-collapse supernova models (Müller \& Janka 1997; Fryer et al. 2004; Scheidegger et al. 2008, 2010).
The total energy radiated in the form of gravitational waves due to nonspherical mass flow is given in the quadrupole approximation by (see, e.g., Misner et al. 1993)

$$
\begin{aligned}
E_{\mathrm{M}}= & \frac{c^{3}}{5 G} \int_{0}^{\infty} \sum_{i j}\left[\frac{\mathrm{d}}{\mathrm{d} t}\left(I_{i j}^{\mathrm{TT}}-\frac{1}{3} \delta_{i j} \sum_{l} I_{l l}^{\mathrm{TT}}\right)\right]^{2} \mathrm{~d} t \\
= & \frac{c^{3}}{15 G} \int_{0}^{\infty} \mathrm{d} t\left[\left(\dot{I}_{x x}^{\mathrm{TT}}-\dot{I}_{y y}^{\mathrm{TT}}\right)^{2}+\left(\dot{I}_{x x}^{\mathrm{TT}}-\dot{I}_{z z}^{\mathrm{TT}}\right)^{2}\right. \\
& \left.+\left(\dot{I}_{y y}^{\mathrm{TT}}-\dot{I}_{z z}^{\mathrm{TT}}\right)^{2}+6\left(\left(\dot{I}_{x y}^{\mathrm{TT}}\right)^{2}+\left(\dot{I}_{x z}^{\mathrm{TT}}\right)^{2}+\left(\dot{I}_{y z}^{\mathrm{TT}}\right)^{2}\right)\right],
\end{aligned}
$$

with $\dot{I}_{i j}^{\mathrm{TT}} \equiv \partial I_{i j}^{\mathrm{TT}} / \partial t$, and the corresponding GW spectral energy density is given by (where $v$ denotes the frequency)

$$
\begin{aligned}
\frac{\mathrm{d} E_{\mathrm{M}}}{\mathrm{d} v}= & \frac{2 c^{3}}{15 G}(2 \pi v)^{2}\left[\left|\widetilde{I}_{x x}^{\mathrm{TT}}-\widetilde{I}_{y y}^{\mathrm{TT}}\right|^{2}+\left|\widetilde{I}_{x x}^{\mathrm{TT}}-\widetilde{I}_{z z}^{\mathrm{TT}}\right|^{2}\right. \\
& \left.+\left|\widetilde{I}_{y y}^{\mathrm{TT}}-\widetilde{I}_{z z}^{\mathrm{TT}}\right|^{2}+6\left(\left|\widetilde{I}_{x y}^{\mathrm{TT}}\right|^{2}+\left|\widetilde{I}_{x z}^{\mathrm{TT}}\right|^{2}+\left|\widetilde{I}_{y z}^{\mathrm{TT}}\right|^{2}\right)\right],
\end{aligned}
$$

where

$\widetilde{I}_{i j}^{\mathrm{TT}}(v)=\int_{-\infty}^{\infty} I_{i j}^{\mathrm{TT}}(t) \mathrm{e}^{-2 \pi \mathrm{i} v t} \mathrm{~d} t$

is the Fourier transform of $I_{i j}^{\mathrm{TT}}(t)$.

\subsubsection{Anisotropic neutrino emission}

To determine the gravitational wave signal associated with the anisotropic emission of neutrinos, we follow Müller \& Janka (1997) and use Eq. (16) of Epstein (1978) in the limit of a distant source, $R \rightarrow \infty$, together with the approximation that the gravitational wave signal measured by an observer at time $t$ is caused only by radiation emitted at time $t^{\prime}=t-R / c$. Hence, we take $t-t^{\prime}=$ const. $=R / c$, i.e., we assume that only the neutrino pulse itself causes a gravitational wave signal, whereas memory effects, which prevail after the pulse has passed the observer, are disregarded.

With these simplifications, the dimensionless gravitational wave amplitudes of the two polarization modes are given in the transverse-traceless gauge for an observer located at a distance $R$ along the $z$-axis of the observer frame by Müller \& Janka (1997)

$h_{+}(t)=\frac{2 G}{c^{4} R} \int_{0}^{t} \mathrm{~d} t^{\prime} \int_{4 \pi} \mathrm{d} \Omega^{\prime}(1+\cos \theta) \cos 2 \phi \frac{\mathrm{d} \Lambda}{\mathrm{d} \Omega^{\prime}}\left(\mathbf{\Omega}^{\prime}, t^{\prime}\right)$

and

$h_{\times}(t)=\frac{2 G}{c^{4} R} \int_{0}^{t} \mathrm{~d} t^{\prime} \int_{4 \pi} \mathrm{d} \Omega^{\prime}(1+\cos \theta) \sin 2 \phi \frac{\mathrm{d} \Lambda}{\mathrm{d} \Omega^{\prime}}\left(\mathbf{\Omega}^{\prime}, t^{\prime}\right)$,

respectively. Here $\mathrm{d} \Lambda\left(\Omega^{\prime}, t^{\prime}\right) / \mathrm{d} \Omega^{\prime}$ is given by Eq. (12) and denotes the total neutrino energy radiated at time $t^{\prime}$ per unit of time into a solid angle $\mathrm{d} \Omega^{\prime}$ in direction $\left(\theta^{\prime}, \phi^{\prime}\right)$. Except for positiondependent factors the gravitational wave amplitudes are simply a function of this quantity provided by the ray-by-ray transport approximation (note that in Müller \& Janka 1997, we used the symbol $L_{v}$ instead of $\Lambda$ ).

The angular integration, $\mathrm{d} \Omega^{\prime}=-\mathrm{d}\left(\cos \theta^{\prime}\right) \mathrm{d} \phi^{\prime}$, in Eqs. (36) and (37) extends over all angles $\theta^{\prime}$ and $\phi^{\prime}$ in the coordinate frame of the source $\left(x^{\prime}, y^{\prime}, z^{\prime}\right)$ that we identify with the (arbitrarily chosen) spherical polar coordinate frame to which the hydrodynamic results were mapped from the Yin-Yang grid employed 

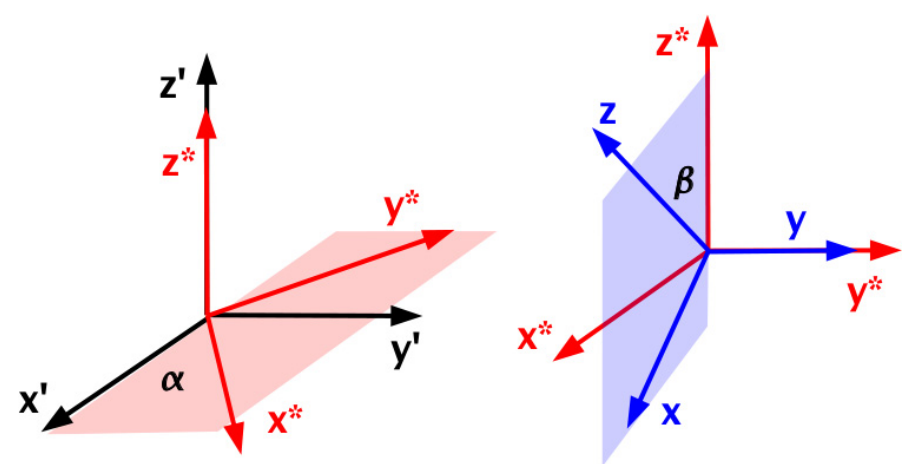

Fig. 10. Relation between the source coordinate system $\left(x^{\prime}, y^{\prime}, z^{\prime}\right)$ and the observer coordinate system $(x, y, z)$. Changing from the observer system to the source system involves a rotation by an angle $\alpha$ about the $z^{\prime}$-axis to an intermediate coordinate system $\left(x^{*}, y^{*}, z^{*}\right)$, followed by a rotation by an angle $\beta$ about the $y^{*}$-axis (which thus is also the $y$-axis).

in the simulations. For the evaluation of the polarisation modes we used the (asymptotic) values of $\mathrm{d} \Lambda(\Omega, t) / \mathrm{d} \Omega$ extracted at a radius of $500 \mathrm{~km}$ from our $3 \mathrm{D}$ models.

The angles $\theta$ and $\phi$ in Eqs. (36) and (37) are measured in the observer frame $(x, y, z)$, while the neutrino luminosity is measured in the source frame $\left(x^{\prime}, y^{\prime}, z^{\prime}\right)$. To allow for an arbitrary orientation of the observer relative to the source, we introduce two viewing angles $\alpha \in[-\pi,+\pi]$ and $\beta \in[0, \pi]$ (see Fig. 10). The coordinates measured in the observer frame are then related to the coordinates in the source frame by the following coordinate transformations

$x^{*}=x^{\prime} \cos \alpha+y^{\prime} \sin \alpha$,

$y^{*}=-x^{\prime} \sin \alpha+y^{\prime} \cos \alpha$,

$z^{*}=z^{\prime}$

and

$x=x^{*} \cos \beta-z^{*} \sin \beta$,

$y=y^{*}$,

$z=x^{*} \sin \beta+z^{*} \cos \beta$.

With these coordinate transformations and the relations

$x=r \sin \theta \cos \phi$,

$y=r \sin \theta \sin \phi$,

$z=r \cos \theta$

between Cartesian coordinates $(x, y, z)$ and spherical polar coordinates $(r, \theta, \phi)$, we obtain

$$
\begin{aligned}
\sin \theta \cos \phi= & \left(\cos \phi^{\prime} \cos \alpha+\sin \phi^{\prime} \sin \alpha\right) \sin \theta^{\prime} \cos \beta \\
& -\cos \theta^{\prime} \sin \beta, \\
\sin \theta \sin \phi= & \left(\sin \phi^{\prime} \cos \alpha-\cos \phi^{\prime} \sin \alpha\right) \sin \theta^{\prime}, \\
\cos \theta= & \left(\cos \phi^{\prime} \cos \alpha+\sin \phi^{\prime} \sin \alpha\right) \sin \theta^{\prime} \sin \beta \\
& +\cos \theta^{\prime} \cos \beta
\end{aligned}
$$

These expressions relate the angular coordinates in the observer frame $(\theta, \phi)$ to those in the source frame $\left(\theta^{\prime}, \phi^{\prime}\right)$. For the special case $\alpha=0$ they were already presented by Kotake et al. (2009a). Using Eq. (49) and the equalities

$$
\begin{aligned}
& \sin 2 \phi=\frac{2 x y}{x^{2}+y^{2}}, \\
& \cos 2 \phi=\frac{x^{2}-y^{2}}{x^{2}+y^{2}}
\end{aligned}
$$

derived from Eqs. (44) to (46), the two polarization modes (Eqs. (36) and (37)) are given by

$h_{\mathrm{S}}(t, \alpha, \beta)=\frac{2 G}{c^{4} R} \int_{0}^{t} \mathrm{~d} t^{\prime} \Lambda\left(t^{\prime}\right) \alpha_{\mathrm{S}}\left(t^{\prime}, \alpha, \beta\right)$,

where $S \in(+, \times)$ and $\Lambda(t)$ is the angular integral of the neutrino energy radiated at time $t$ per unit of time given in Eqs. (13) and (15).

$\alpha_{\mathrm{S}}(t, \alpha, \beta)=\frac{1}{\Lambda(t)} \int_{4 \pi} \mathrm{d} \Omega^{\prime} W_{\mathrm{S}}\left(\Omega^{\prime}, \alpha, \beta\right) \frac{\mathrm{d} \Lambda}{\mathrm{d} \Omega^{\prime}}\left(\mathbf{\Omega}^{\prime}, t\right)$,

are anisotropy parameters, which provide a quantitative measure of the time-dependent anisotropy of the emission in both polarization modes. Note that the evaluation of the anisotropy parameter $\alpha(t)$ defined in Eq. (29) of Müller \& Janka (1997), which should not be confused with the observer angle $\alpha$ introduced in Fig. 10, does neither involve a dependence on observer angles $(\alpha, \beta)$ nor on the polarization mode.

The angular weight functions appearing in the above expression for the anisotropy parameters are given by

$W_{\mathrm{S}}\left(\theta^{\prime}, \phi^{\prime}, \alpha, \beta\right)=\frac{D_{\mathrm{S}}\left(\theta^{\prime}, \phi^{\prime}, \alpha, \beta\right)}{N\left(\theta^{\prime}, \phi^{\prime}, \alpha, \beta\right)}$,

where

$$
\begin{aligned}
D_{+}= & {\left[1+\left(\cos \phi^{\prime} \cos \alpha+\sin \phi^{\prime} \sin \alpha\right) \sin \theta^{\prime} \sin \beta+\cos \theta^{\prime}\right.} \\
& \cos \beta]\left\{\left[\left(\cos \phi^{\prime} \cos \alpha+\sin \phi^{\prime} \sin \alpha\right) \sin \theta^{\prime} \cos \beta\right.\right. \\
& \left.\left.-\cos \theta^{\prime} \sin \beta\right]^{2}-\sin ^{2} \theta^{\prime}\left(\sin \phi^{\prime} \cos \alpha-\cos \phi^{\prime} \sin \alpha\right)^{2}\right\}, \\
D_{\times}= & {\left[1+\left(\cos \phi^{\prime} \cos \alpha+\sin \phi^{\prime} \sin \alpha\right) \sin \theta^{\prime} \sin \beta+\cos \theta^{\prime}\right.} \\
& \cos \beta] 2\left[\left(\cos \phi^{\prime} \cos \alpha+\sin \phi^{\prime} \sin \alpha\right) \sin \theta^{\prime} \cos \beta\right. \\
& \left.-\cos \theta^{\prime} \sin \beta\right] \sin \theta^{\prime}\left(\sin \phi^{\prime} \cos \alpha-\cos \phi^{\prime} \sin \alpha\right), \\
N= & {\left[\left(\cos \phi^{\prime} \cos \alpha+\sin \phi^{\prime} \sin \alpha\right) \sin \theta^{\prime} \cos \beta-\cos \theta^{\prime}\right.} \\
& \sin \beta]^{2}+\sin ^{2} \theta^{\prime}\left(\sin \phi^{\prime} \cos \alpha-\cos \phi^{\prime} \sin \alpha\right)^{2} .
\end{aligned}
$$

Choosing $\alpha=0$ and $\beta=\pi / 2$ the observer is located in the equatorial plane of the source (i.e., perpendicular to the source's $z^{\prime}$-axis) at the azimuthal position $\phi^{\prime}=0$. In that case one obtains simpler expressions for the angular functions (see also Kotake et al. 2009a)

$$
\begin{aligned}
& \left.W_{+}\right|_{\mathrm{e}}=\left(\cos ^{2} \theta^{\prime}-\sin ^{2} \theta^{\prime} \sin ^{2} \phi\right) \frac{1+\sin \theta^{\prime} \cos \phi^{\prime}}{\cos ^{2} \theta^{\prime}+\sin ^{2} \theta^{\prime} \sin ^{2} \phi^{\prime}}, \\
& \left.W_{\times}\right|_{\mathrm{e}}=-2 \cos \theta^{\prime} \sin \theta^{\prime} \sin \phi^{\prime} \frac{1+\sin \theta^{\prime} \cos \phi^{\prime}}{\cos ^{2} \theta^{\prime}+\sin ^{2} \theta^{\prime} \sin ^{2} \phi^{\prime}} .
\end{aligned}
$$

Note that for axisymmetric sources $h_{\times}=0$.

In general, the total energy $E_{\mathrm{GW}}(t)$ radiated to infinity by a source in form of gravitational waves until time $t$ is given by (see, e.g., Misner et al. 1993; Greek indices run from 0 to 3, and repeated indices are summed over)

$E_{\mathrm{GW}}(t)=\int_{0}^{t} \mathrm{~d} t^{\prime} \int_{S_{\infty}^{2}} \tau_{0 v} n^{v} r^{2} \mathrm{~d} \Omega$,

where the angular integration is performed over a two-sphere at spatial infinity $S_{\infty}^{2}$, and $n^{\mu}=(0,1,0,0)$ is a unit spacelike vector in polar coordinates $\{c t, r, \theta, \phi\}$ normal to $S_{\infty}^{2}$. Denoting by $\langle\ldots\rangle$ an average over several wavelengths, the gravitational-wave energy-momentum tensor $\tau_{\mu \nu}$ is given in transverse-traceless gauge by

$\tau_{\mu \nu}=\frac{c^{5}}{32 \pi G}\left\langle\left(\partial_{\mu} h_{\rho \sigma}^{\mathrm{TT}}\right)\left(\partial_{\nu} h_{\mathrm{TT}}^{\rho \sigma}\right)\right\rangle$. 
Thus, Eq. (60) can be rewritten as

$E_{\mathrm{GW}}(t)=\frac{c^{3}}{32 \pi G} \int_{0}^{t} \mathrm{~d} t^{\prime} \int_{S_{\infty}^{2}} r^{2} \mathrm{~d} \Omega\left\langle\left(\partial_{t} h_{i k}^{\mathrm{TT}}\right)\left(\partial_{r} h_{\mathrm{TT}}^{i k}\right)\right\rangle$,

where we have used the facts that $h_{0 v}^{\mathrm{TT}}=0, h_{i, r}^{\mathrm{TT}}=0$, and $c \partial_{r} h_{i k}^{\mathrm{TT}}=-\partial_{t} h_{i k}^{\mathrm{TT}}$ for radially outgoing gravitational radiation. Evaluating the double sum in Eq. (62) and using the relations $h_{\theta \theta}^{\mathrm{TT}}=-h_{\phi \phi}^{\mathrm{TT}}=h_{+}$and $h_{\theta \phi}^{\mathrm{TT}}=h_{\phi \theta}^{\mathrm{TT}}=h_{\times}$(see, e.g., Misner et al. 1993), we finally find

$E_{\mathrm{GW}}(t)=\frac{c^{3}}{16 \pi G} \int_{0}^{t} \mathrm{~d} t^{\prime} \int_{S_{\infty}^{2}} r^{2} \mathrm{~d} \Omega\left\langle\left(\partial_{t} h_{+}\right)^{2}+\left(\partial_{t} h_{\times}\right)^{2}\right\rangle$.

Inserting the expressions for $h_{+}$and $h_{\times}$given in Eqs. (52) into (63), we obtain for the energy $E_{\mathrm{N}}(t)$ radiated in form of gravitational waves until time $t$ due to anisotropic neutrino emission

$E_{\mathrm{N}}(t)=\frac{G}{4 \pi c^{5}} \int_{0}^{t} \mathrm{~d} t^{\prime} \int_{4 \pi} \mathrm{d} \Omega_{\alpha \beta}\left[l_{+}^{2}\left(t^{\prime}, \alpha, \beta\right)+l_{\times}^{2}\left(t^{\prime}, \alpha, \beta\right)\right]$

with $\mathrm{d} \Omega_{\alpha \beta}=\sin \beta \mathrm{d} \beta \mathrm{d} \alpha$ and

$l_{\mathrm{S}}(t, \alpha, \beta)=\Lambda(t) \alpha_{\mathrm{S}}(t, \alpha, \beta)$.

The corresponding spectral energy density is given by

$\frac{\mathrm{d} E_{\mathrm{N}}}{\mathrm{d} v}=\frac{G}{2 \pi c^{5}}|\tilde{l}(v)|^{2}$

where $\tilde{l}(v)$ is the Fourier transform of

$l(t)=\left\{\int_{4 \pi} \mathrm{d} \Omega_{\alpha \beta}\left[l_{+}^{2}(t, \alpha, \beta)+l_{\times}^{2}(t, \alpha, \beta)\right]\right\}^{1 / 2}$.

For completeness we also provide an expression for the total energy radiated in form of gravitational waves until time $t$, i.e., due to anisotropic mass flow and neutrino emission. It is obtained by inserting the total GW amplitude, i.e., the sum of the amplitudes given by Eqs. (19) and (52) into (63), which leads to

$$
\begin{aligned}
E_{\mathrm{GW}}= & \frac{c^{3}}{16 \pi G} \int_{0}^{t} \mathrm{~d} t^{\prime} \\
& \times \int_{4 \pi} \mathrm{d} \Omega\left[\left(\frac{2 G}{c^{4}} l_{+}+\partial_{t} A_{+}\right)^{2}+\left(\frac{2 G}{c^{4}} l_{\times}+\partial_{t} A_{\times}\right)^{2}\right] .
\end{aligned}
$$

\subsection{Results}

Although an observer can only measure the total gravitational wave amplitude, i.e., that due to the combined effect of nonradial flow and anisotropic neutrino emission, we will first discuss the GW signal of non-radial mass flow only, because it reflects the various phases of the post-bounce evolution already introduced in the discussion of the neutrino signal above.

Until post-shock convection and the SASI are eventually mature at around $150 \mathrm{~ms}$, the GW signal is very small (Fig. 11). Note that our models are not able to follow the GW emission that is caused by prompt post-shock convection because of the excised inner region of the PNS (Marek et al. 2009). Later on, sizable g-mode activity is instigated in the outer layers of the proto-neutron star by convective overturn and the SASI during the hydrodynamically vigorous pre-explosion phase, and by the impact of anisotropic accretion flows during the subsequent post-explosion accretion phase (Marek et al. 2009). This g-mode activity is the cause of GW signals (Marek et al. 2009; Murphy et al. 2009; Yakunin et al. 2010), whose maximum amplitudes are on the order of a few centimeters centered around zero.

The GW frequency distribution possesses a very broad maximum in the range of $100 \mathrm{~Hz}$ to $500 \mathrm{~Hz}$, and the frequency corresponding to this maximum slowly increases with time (Fig. 11). Partially already during the post-explosion accretion phase, but at latest when the shock wave starts to rapidly propagate to large radii between $\sim 0.4 \mathrm{~s}$ and $\sim 0.7 \mathrm{~s}$ (see Figs. 3 and 4 ), the GW amplitudes start to grow by about a factor of ten until approximately asymptoting at $\sim 0.9 \mathrm{~s}$ in the case of the models based on the progenitor W15, and at $\sim 1 \mathrm{~s}$ in the case of models based on the progenitor L15, respectively (Fig. 11). This growth of the amplitude is associated with the anisotropic expansion of the shock wave, and a positive/negative wave amplitude indicates a prolate/oblate explosion, respectively (Murphy et al. 2009).

While the GW amplitudes grow, the GW energy distribution $\mathrm{d} E_{\mathrm{M}} / \mathrm{d} v$ becomes narrower and dimmer, and the frequency at maximum power continues to increase. The latter effect was also observed in the 2D models of Murphy et al. (2009). At late times, the GW signal of the W15 models clearly signifies the convective activity inside the proto-neutron star through low-amplitude, high-frequency fluctuations around the asymptotically roughly constant mean GW amplitudes, while no such fluctuations are present in the case of the L15 models (see discussion of the neutrino signal above). This model discrepancy is also evident from the energy spectrograms, which do exhibit a pronounced broad maximum (between $\sim 350 \mathrm{~Hz}$ and $\sim 550 \mathrm{~Hz}$ ) at $t>0.8 \mathrm{~s}$ in the case of the W15 models, but none for the L15 ones. Furthermore note that until the end of the simulations the frequency of the maximum of $\mathrm{d} E_{\mathrm{M}} / \mathrm{d} v$ has increased from around $100 \mathrm{~Hz}$ to almost $500 \mathrm{~Hz}$ for the former models (owing to the increasing speeds of mass motions in the postshock region at times $\lesssim 0.5 \mathrm{~s}$ and because of the increased compactness of the proto-neutron star at times $\gtrsim 0.6 \mathrm{~s}$, respectively).

The behavior of the total (matter plus neutrinos) GW amplitudes is significantly different from that of the flow-only GW amplitudes for models that exhibit PNS convection below the neutrinosphere, i.e., for the models based on the progenitors W15 and N20. Particularly at late times, anisotropic neutrino emission causes a continuing growth of the GW amplitudes (instead of a saturation) in these models, while this is not the case for the L15 models (see Fig. 12, and compare with Fig. 11). The latter behavior is also reflected in the time evolution of the asymmetry parameter $\alpha_{\mathrm{S}}$ (Eq. (53)) of the neutrino emission (Fig. 13). The asymmetry parameter is practically zero in model L15-3 at late times, while it remains, after having temporarily grown to values beyond about $0.4-0.5 \%$, at the level of $\sim 0.3 \%$ until the end of the simulations in models W15-4 and N20-2.

The final GW amplitudes are up to a factor of two to three higher when taking the contribution of anisotropic neutrino emission into account. In contrast, the amount of energy radiated in the form of $\mathrm{GW}$, which is proportional to the third timederivative of the quadrupole moment and hence proportional to the time derivative of the GW amplitude is only insignificantly changed, and is practically constant for all simulated models after the onset of the explosion (see Fig. 14). The integral value of the GW energy radiated by neutrinos is low $(\$ 1 \%)$ compared to that emitted by matter through the slow variation of the GW neutrino amplitude with time, i.e., its time derivative is much smaller than that of the GW matter amplitude. For this reason we also abstained from evaluating the total energy radiated in form of GW (Eq. (68)). It differs little from that caused by anisotropic matter flow alone (Eq. (33)), because the mixed term in Eq. (68), 

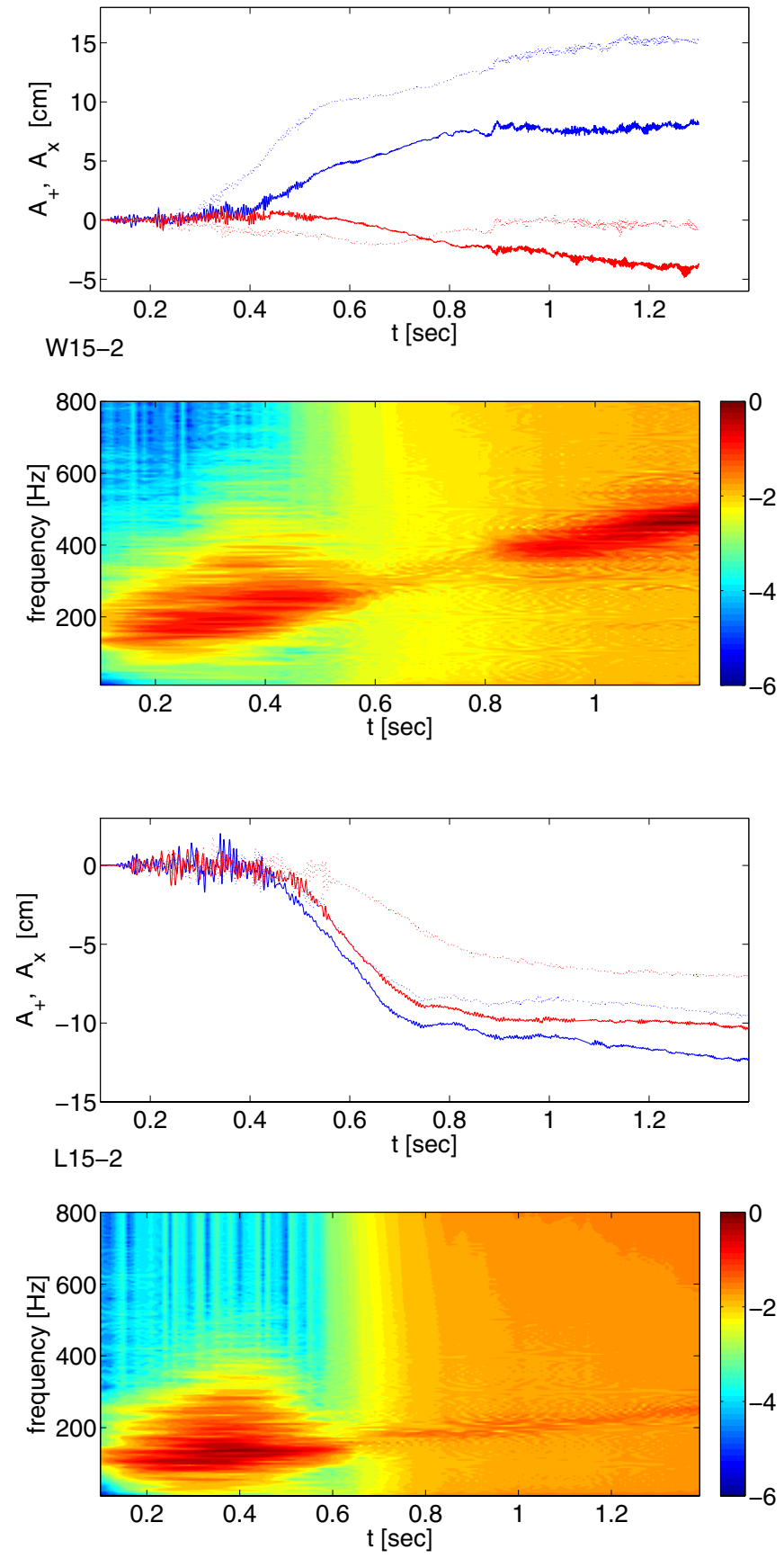
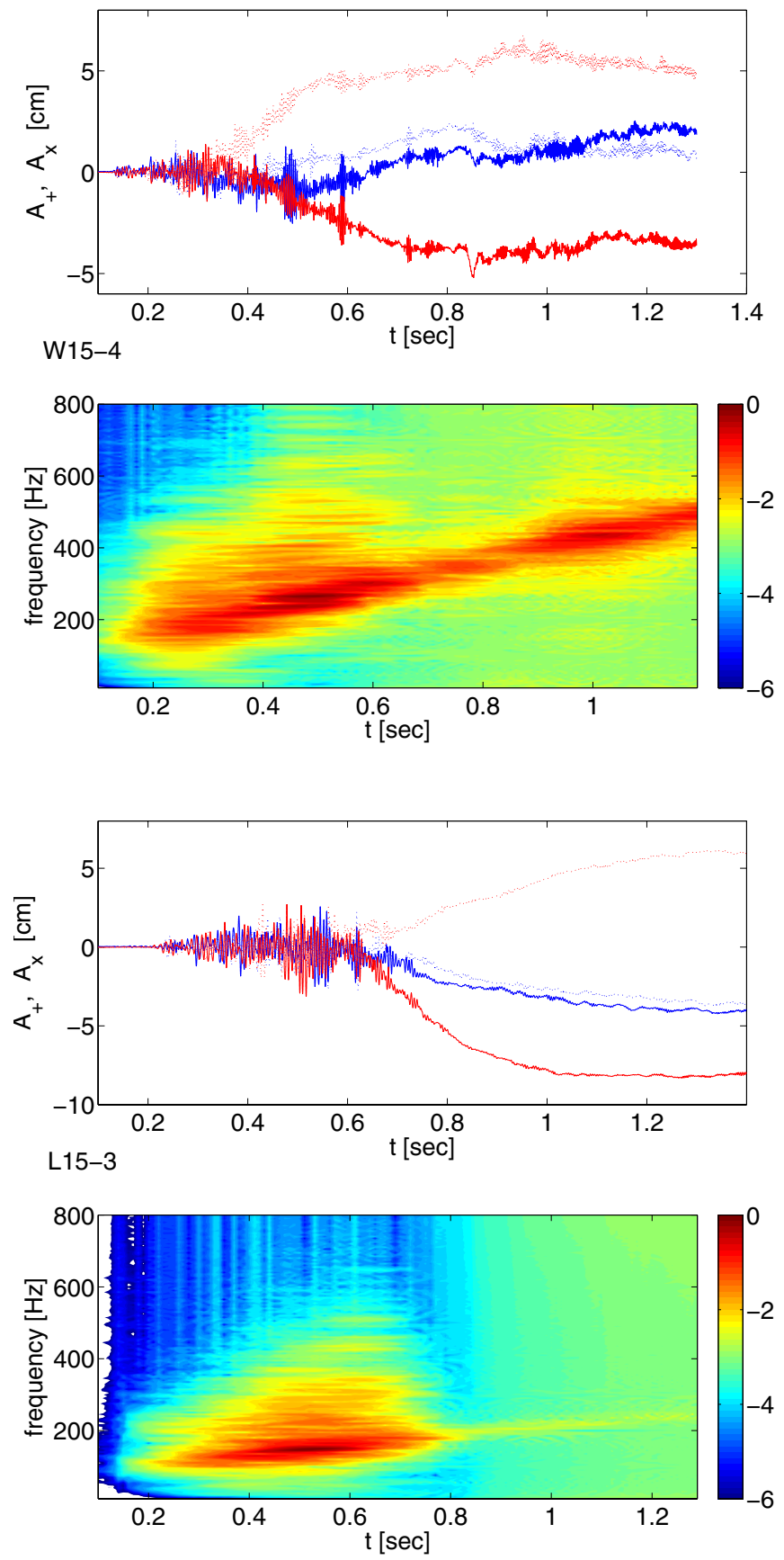

Fig. 11. The four panels show the gravitational wave amplitudes (top) and spectrograms of $\mathrm{d} E_{\mathrm{M}} / \mathrm{d} v$ (bottom; normalized to the absolute maximum) arising from non-spherical mass flow of models W15-2 (top left), W15-4 (top right), L15-2 (bottom left), and L15-3 (bottom right), respectively. Blue curves give the amplitude $A_{+}$at the pole (solid) and the equator (dotted), while red curves show the other independent mode of polarization $A_{\times}$ from the same directions.

resulting from the square of the sum of the matter and neutrino parts, contributes $\$ 10 \%$ to the total radiated GW energy, and the pure neutrino term $\lesssim 1 \%$. Figure 14 also shows that the (small) contribution of anisotropic neutrino emission to the radiated GW energy is enhanced at late times when proto-neutron star convection occurs below the neutrinosphere, as it is the case for models W15-4 and in particular N20-2.

The variation of the total GW amplitudes with observer angle is illustrated in Fig. 15 for model W15-4 at $1.3 \mathrm{~s}$ (when the simulation was stopped). Both the amplitude variations and the typical angular size of the speckled GW emission are similar for all other simulated models. The model-independent level of the amplitude variations is also supported by Fig. 12 when comparing various amplitudes at any given (late) time.

The (normalized) amplitude spectrograms of the total gravitational wave amplitudes $\left(\mathrm{d}\left(A_{+, \times}+R h_{+, x}\right) / \mathrm{d} v\right.$; Figs. 16 and 17) illustrate two model-independent findings. Firstly, during the hydrodynamically vigorous pre-explosion and post-explosion accretion phases $(0.2 \lesssim t \lesssim 0.5-0.7 \mathrm{~s})$ the spectra of all models are characterized by some power at low frequencies $(\$ 100 \mathrm{~Hz})$ and a broad power maximum at frequencies $\sim 200 \mathrm{~Hz}$ and another weak one at $\sim 800 \mathrm{~Hz}$. The latter broad maximum at high 

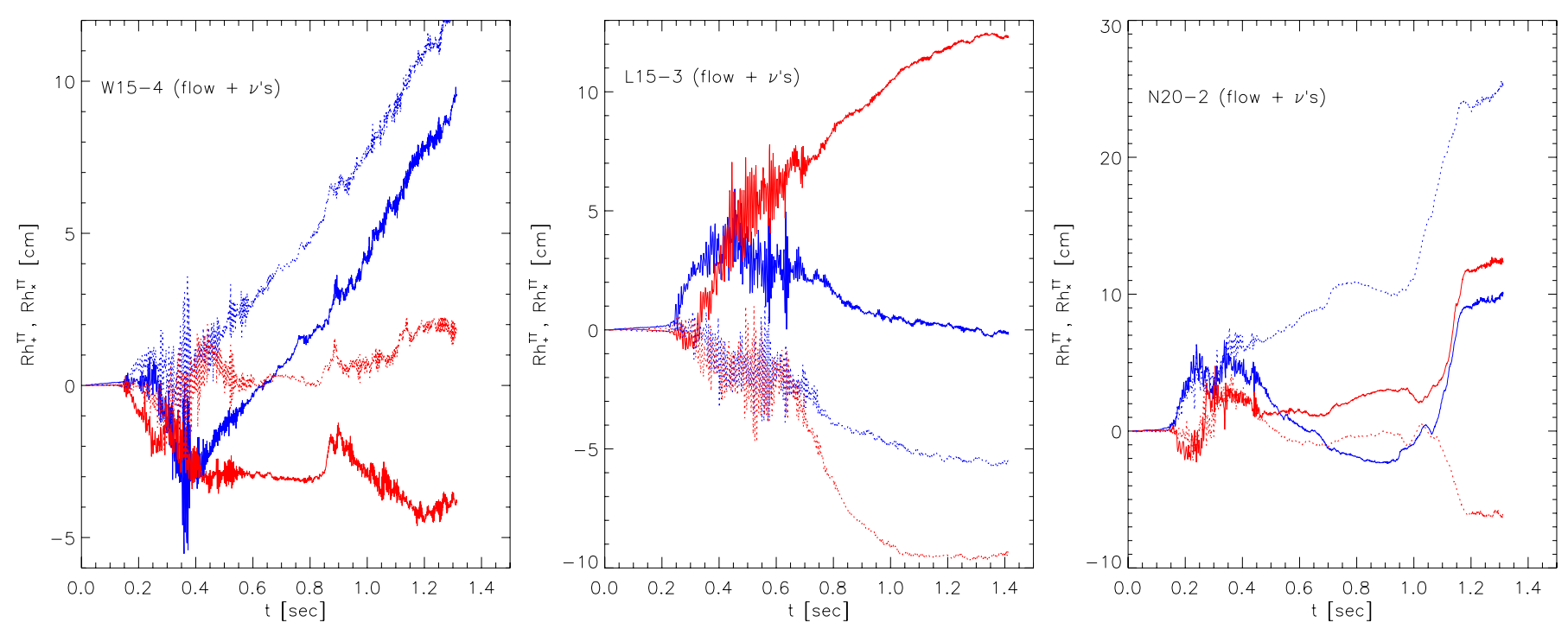

Fig. 12. Gravitational wave amplitudes $R h_{+}^{\mathrm{TT}}$ (blue) and $R h_{\times}^{\mathrm{TT}}$ (red) due to anisotropic mass flow and neutrino emission as a function of time for models W15-4 (left), L15-3 (middle), and N20-3 (right), respectively. The solid curves show the amplitudes for an observer located above the north pole ( $\alpha=\beta=0$; see Fig. 10) of the source, while the other curves give the amplitudes at the equator $(\alpha=0, \beta=\pi / 2)$.
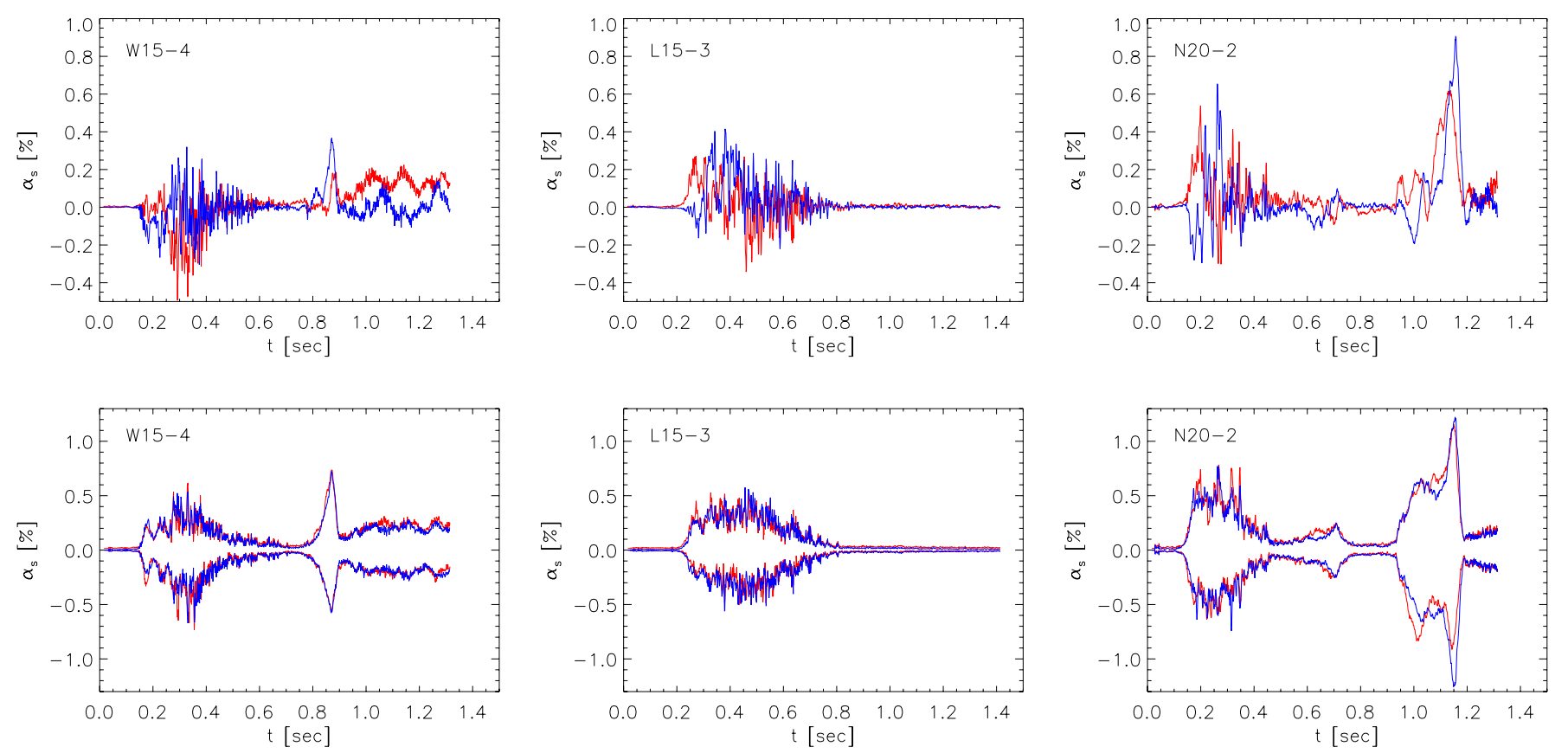

Fig. 13. Asymmetry parameter $\alpha_{S}$ of the neutrino emission (Eq. (53)) as a function of time for models W15-4 (left), L15-3 (middle), and N20-2 (right), respectively. The panels in the upper row show $\alpha_{+}$(blue) and $\alpha_{\times}$(red) for a particular observer direction, while the panels in the lower row give for both parameters the maximum and minimum values in all directions.

frequency is more pronounced in the models based on the W15 and N20 progenitors and in the cross polarization GW mode. Secondly, during the post-accretion phase $(t \gtrsim 0.7 \mathrm{~s})$ the spectra of all models are dominated by a low-frequency $(\$ 40 \mathrm{~Hz})$ contribution peaked toward the lower end of the spectrogram. In the models where PNS convection occurs below the neutrinosphere (models W15 and N20) we also find a doublepeaked high-frequency contribution decreasing/increasing from $\sim 700 \mathrm{~Hz}(400 \mathrm{~Hz})$ at $t \sim 0.8 \mathrm{~s}$, and eventually merging into a single power maximum at $\sim 500 \mathrm{~Hz}$ at $t \sim 1.2 \mathrm{~s}$. Again, this contribution is more pronounced for the cross polarization GW mode.

The spectra of the total GW amplitudes are dominated by the contribution from non-isotropic neutrino emission at low $(\leq 100 \mathrm{~Hz})$ frequencies (Figs. 16 and 17). At higher frequencies $(\geq 100 \mathrm{~Hz})$ the spectra of model W15-4 show two pronounced maxima (at $100-200 \mathrm{~Hz}$ and $600-800 \mathrm{~Hz}$, respectively) at all times. These maxima are also present in model L15-3 at times $\leq 0.7 \mathrm{~s}$, the high-frequency one being, however, much less pronounced. The lower maximum (at 100-200 Hz) results from g-mode activity in the PNS surface instigated by non-radial flow (SASI, accretion) in the post-shock region until $\sim 0.5-0.7 \mathrm{~s}$. At later times PNS convection is responsible for the peak between 300 and $500 \mathrm{~Hz}$. We have proposed this explanation already for the corresponding maxima present in the GW energy spectrograms arising from non-spherical mass flow (Fig. 11), and discussed why the frequencies of these maxima 
E. Müller et al.: Parametrized 3D models of neutrino-driven supernova explosions
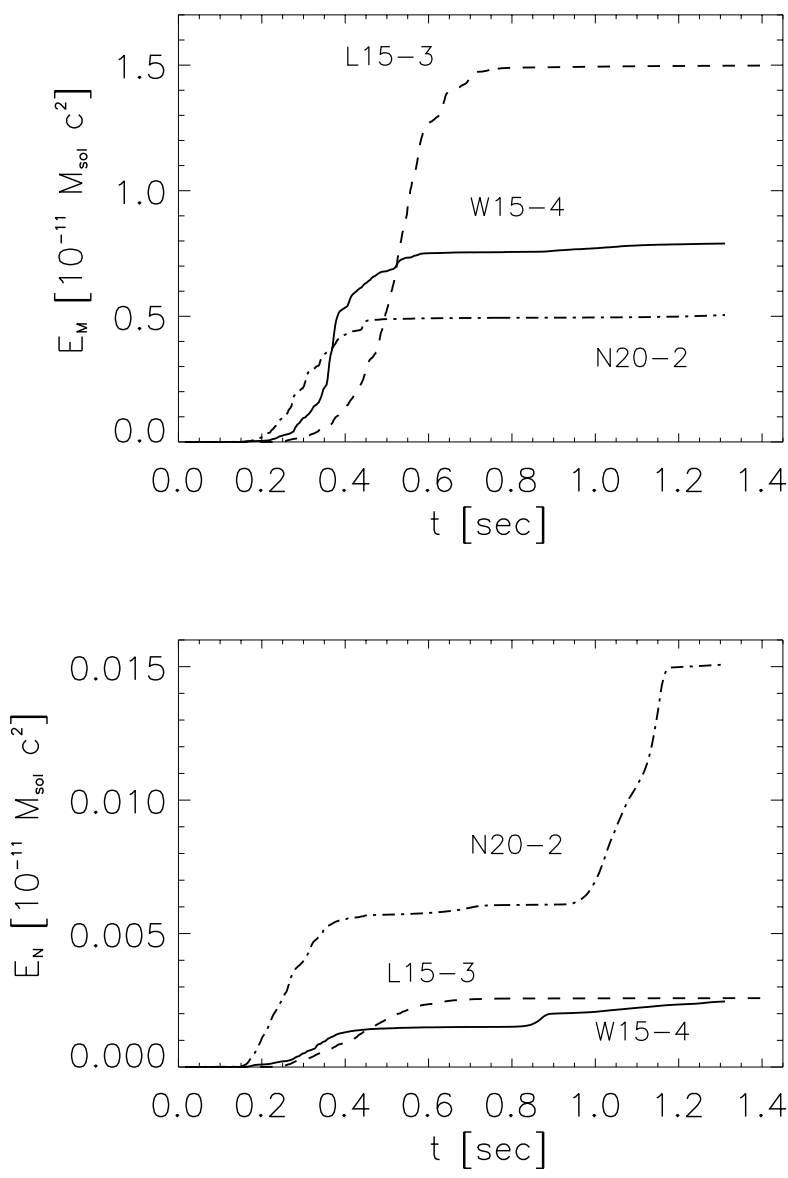

Fig. 14. Energy emitted in form of gravitational waves due to anisotropic mass flow (top panel) and due to anisotropic neutrino emission (bottom panel) as a function of time for models W15-4 (solid), L15-3 (dashed), and N20-2 (dash-dotted), respectively.

increase with time. The source of the high-frequency maximum $(600-800 \mathrm{~Hz})$ is unclear, but a further detailed analysis shows that (i) the maximum is solely caused by non-radial gas flow, i.e., it is not connected to neutrinos; (ii) it does not result from stellar layers below the neutrino sphere but from those close to or slightly above it; and (iii) does not depend on the position of the observer.

Note that the high-frequency maximum present in the amplitude spectrograms is strongly suppressed in the corresponding energy spectrograms (Fig. 11), because the latter involve the squared time derivatives of the amplitudes. Thus, the already high ratio of the low- and high-frequency maxima in the amplitude spectrograms (about two orders of magnitude) translates into an even higher ratio for the energy spectrogram maxima, rendering the high-frequency maximum practically invisible.

\section{Discussion and conclusions}

Based on a set of three-dimensional (3D) parametrized neutrinodriven supernova explosion models of non-rotating 15 and $20 M_{\odot}$ stars, employing a neutrino transport description with a gray spectral treatment and a ray-by-ray approximation of multi-dimensional effects (the scheme is applicable in the regime outside the dense neutron star core, i.e., around and outside the neutrinosphere), we evaluated both the time-dependent and direction-dependent neutrino and gravitational-wave emission of these models. To this end we presented the formalism necessary

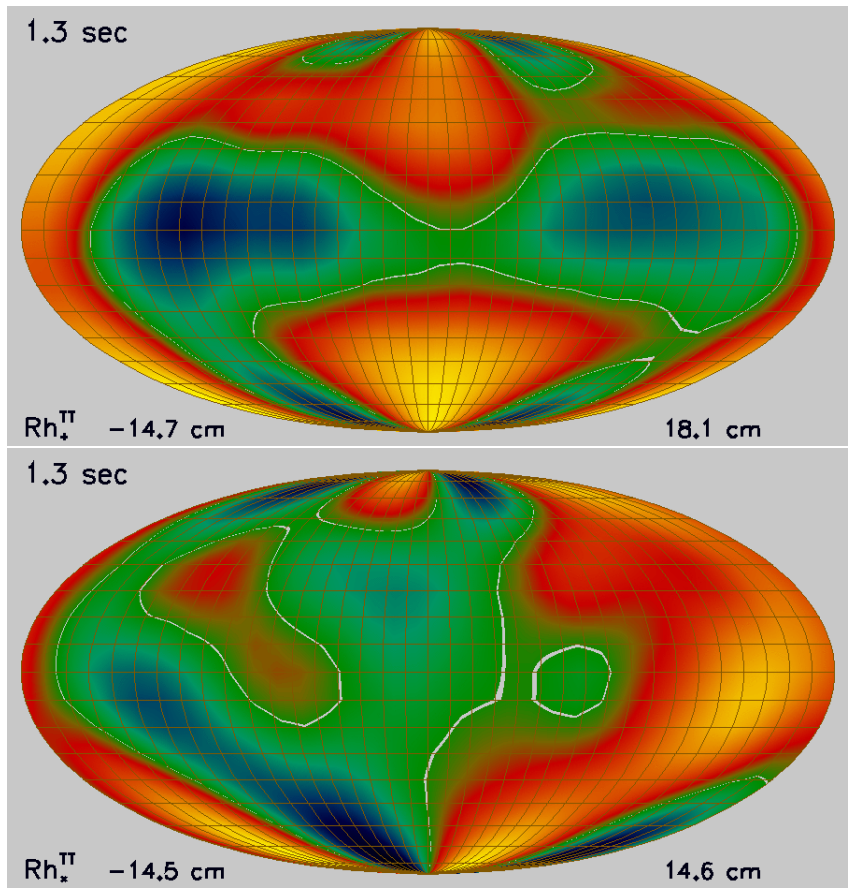

Fig. 15. Gravitational wave amplitudes due to anisotropic mass flow and neutrino emission, $R h_{+}^{\mathrm{TT}}$ (top) and $R h_{\times}^{\mathrm{TT}}$ (bottom), as functions of the observer angles (see Fig. 10) for model W15-4 at $1.3 \mathrm{~s}$ past bounce. The white contours give the locations, where the amplitudes are zero. Yellow and red areas indicate positive amplitudes, green and blue negative ones.

to compute both the observable neutrino and gravitational wave signals for a three-dimensional, spherical source. For the neutrino signal we presented formulas that allow one to estimate the apparent luminosity when the local flux density $F$ on a sphere is known and the ansatz of Eq. (4) about the angular distribution of the intensity can be made. While in general the location of the neutrino-decoupling surface has to be suitably defined (e.g., by a criterion based on the optical depth), our ray-by-ray transport scheme implies that $r^{2} F=$ const. in the free-streaming limit in every direction. Thus, it spares us such a definition of the neutrinosphere for evaluating the angular integration for the observable luminosity. Concerning the gravitational-wave analysis, we extended and generalized previous studies, where the source was either assumed to be axisymmetric or where the formulas for the signals of a 3D source were only given for special observer directions.

Our models followed the evolution from shortly after core bounce up to more than one second into the early cooling evolution of the PNS without imposing any symmetry restrictions and covering a full sphere. The extension over such a relatively long evolution time in 3D was possible through the usage of an axis-free overset grid (the Yin-Yang grid) in spherical polar coordinates, which considerably eases the CFL time-step restriction and avoids axis artifacts. A central region, the dense inner core of the proto-neutron star, was excised from the computational domain and replaced by an inner, time-dependent radial boundary condition and a gravitating point mass at the coordinate origin. Explosions in the models were initiated by neutrino heating at a rate that depends on suitably chosen values of the neutrino luminosities imposed at the inner radial boundary.

The post-bounce evolution of our models can be divided into four distinct phases (Fig. 3). The first phase, the quasispherical shock-expansion phase, lasts from shock formation shortly after core bounce to $80-150 \mathrm{~ms}$, when convection sets in. 


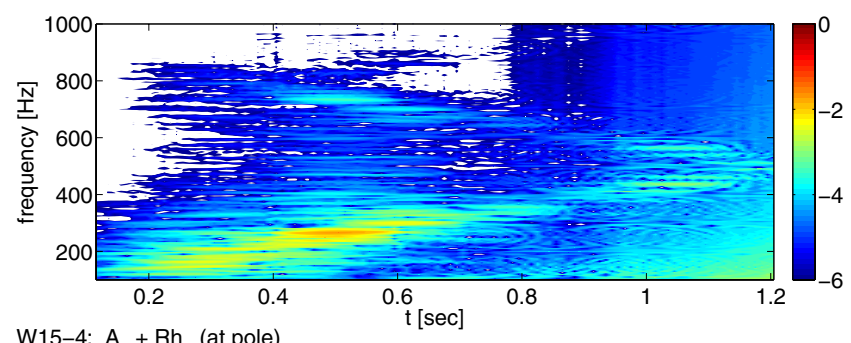

W15-4: $A_{+}+R_{+}($at pole $)$

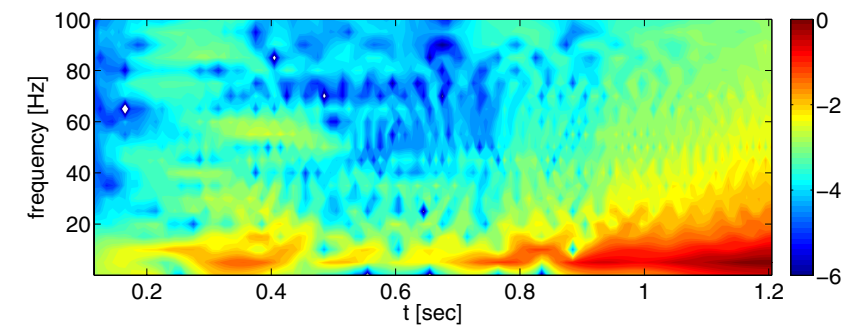

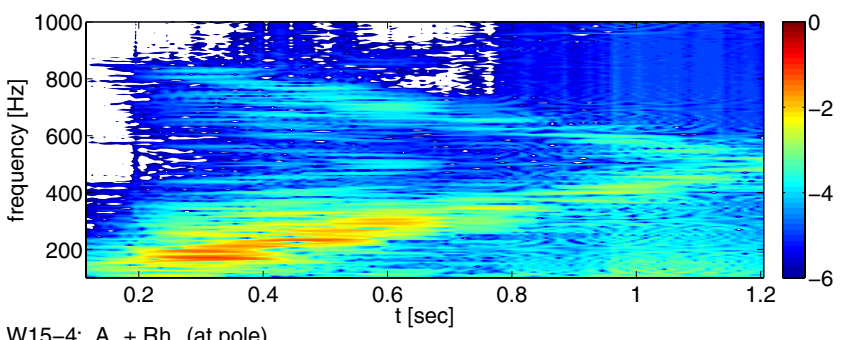

W15-4: $A_{x}+R h_{x}($ at pole $)$

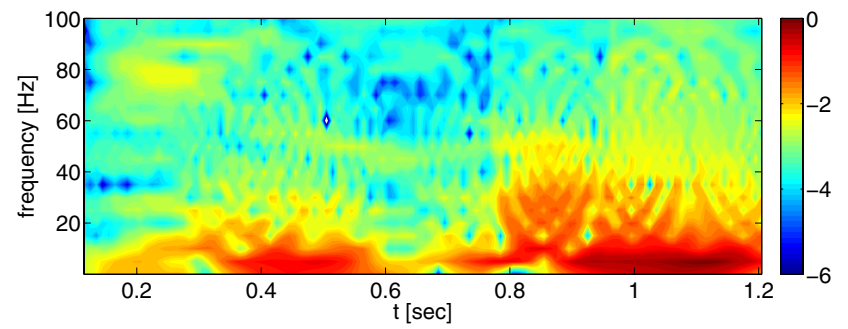

Fig. 16. Normalized (to the absolute maximum) amplitude spectrograms of the total gravitational wave amplitudes $A_{+}+R h_{+}$(left panels) and $A_{\times}+R h_{\times}$(right panels) at the pole for model W15-4. The lower panels show the spectrograms in the frequency range $5 \mathrm{~Hz}$ to $100 \mathrm{~Hz}$, and the upper ones in the frequency range $100 \mathrm{~Hz}$ to $1 \mathrm{kHz}$.

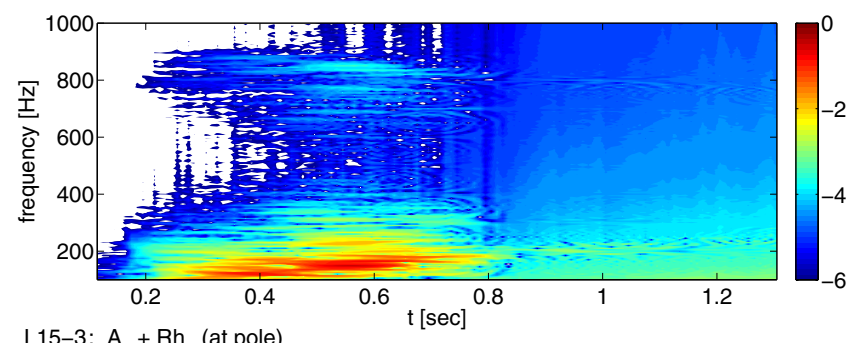

L15-3: $\mathrm{A}_{+}+\mathrm{Rh}_{+}($at pole $)$

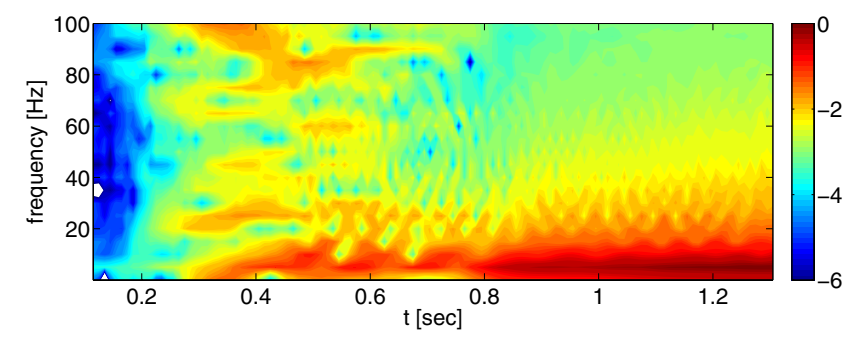

Fig. 17. Same as Fig. 16, but for model L15-3.

The second phase, the hydrodynamically vigorous pre-explosion phase, comprises the growth of post-shock convection and of the standing accretion shock instability (SASI). The post-explosion accretion phase begins when energy deposition by $v$-heating in the post-shock layers becomes sufficiently strong so that the total energy in the post-shock region ultimately becomes positive. During this phase the shock accelerates outward while gas is still accreted onto the PNS. This process is commonly called "shock revival". The duration of the latter two phases depends on the progenitor. During the post-accretion phase, the fourth and final phase characterizing the evolution of our models, accretion ends and the proto-neutron star develops a nearly spherical neutrinodriven wind.

The neutrino emission properties (fluxes and effective spectral temperatures) of our 3D models exhibit the generic timedependent features already known from 2D (axisymmetric) models (e.g., Buras et al. 2006; Scheck et al. 2006; Marek et al. 2009; Brandt et al. 2011), showing fluctuations over the neutron star surface on different spatial and temporal scales. We found that non-radial mass motions caused by the SASI and convection in the neutrino-heated hot-bubble region as well

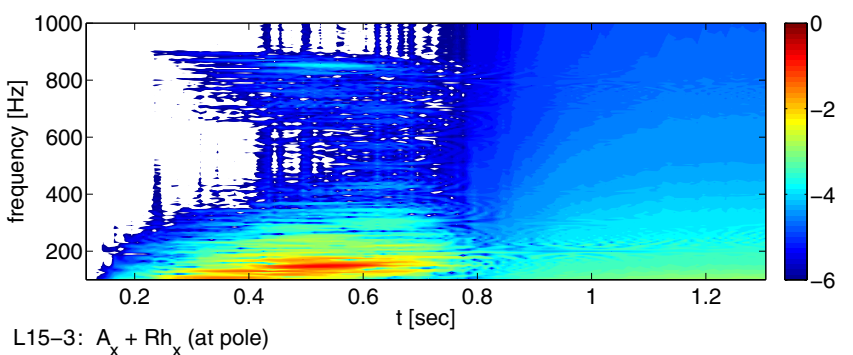

L15-3: $A_{x}+R h_{x}($ at pole $)$

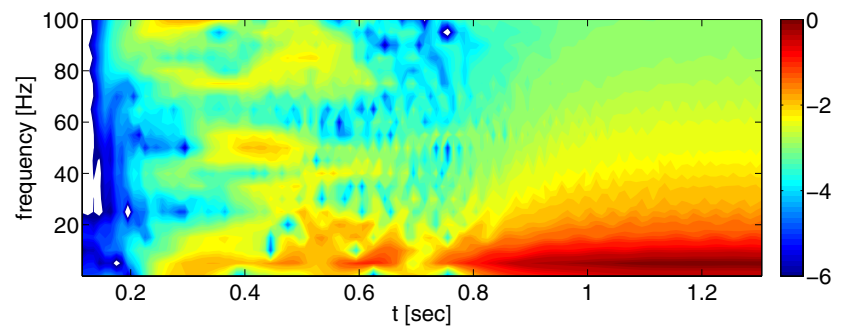

as by PNS convection below the neutrinosphere give rise to a time-dependent, anisotropic emission of neutrinos, particularly of electron neutrinos and anti-neutrinos, and thus also to the emission of gravitational waves. Because very prominent, quasi-periodic sloshing motions of the shock due to the standing accretion-shock instability as visible in 2D simulations are absent and the emission from different surface areas facing an observer adds up incoherently, the modulation amplitudes of the measurable neutrino luminosities and mean energies are significantly lower than predicted by $2 \mathrm{D}$ models (for $2 \mathrm{D}$ results see Marek et al. 2009; Brandt et al. 2011).

During the quasi-spherical shock expansion phase shortly after bounce the level of temporal and angular fluctuations of the neutrino emission is low $\left(\lesssim 10^{-2}\right)$. In contrast, the fluctuation amplitudes reach a level of several $10 \%$ of the average values during the hydrodynamically vigorous pre-explosion phase and the post-explosion accretion phase, where a few distinct, highly time-variable regions or even short-lived single spots with an angular size of $10^{\circ}$ to $20^{\circ}$ are responsible for the brightest emission maxima. As the outward shock expansion is well on its way in the post-explosion accretion phase, still existing 
accretion downdrafts can be responsible for similar fluctuations in the neutrino emission, though the number of corresponding hot spots decreases with diminishing accretion. When accretion has ended and the post-accretion phase has started, directional variations can be caused by the occurrence of Ledoux convection in the outer layers of the proto-neutron star, which we indeed observe in models based on two of our three progenitors (see also the discussion of the influence of the inner radial boundary condition below). The temporal and angular variations of the emission in different directions are even more pronounced when considering the energy flux of the electron neutrinos or electron anti-neutrinos alone (instead of the emission in all neutrino flavors). In that case the angular variations of local flux densities can exceed $100 \%$ in all models during the pre-explosion and post-explosion accretion phases, and the peak values can be close to $200 \%$ during short episodes. The total energy loss rates in neutrinos and the observable luminosities as surfaceintegrated quantities, however, are much smoother in time during all phases, showing fluctuation amplitudes of at most several percent.

The gravitational wave emission also exhibits the generic time-dependent features already known from 2D (axisymmetric) models, but the $3 \mathrm{D}$ wave amplitudes are considerably lower (by a factor of 2-3) than those predicted by 2D models (Müller et al. 2004; Marek et al. 2009; Murphy et al. 2009; Yakunin et al. 2010) owing to less coherent mass motions and neutrino emission. Note in this respect that the GW quadrupole amplitudes, which are usually quoted for $2 \mathrm{D}$ models $\left(A_{20}^{\mathrm{E} 2}\right)$, have to be multiplied by a geometric factor $\sin ^{2} \theta \sqrt{15 / \pi} / 8$ (which is equal to $\approx 0.27$ for $\theta=90^{\circ}$ ). Violent, non-radial hydrodynamic mass motions in the accretion layer and their interaction with the outer layers of the proto-neutron star give rise to a $\mathrm{GW}$ signal with an amplitude of $\sim 2-4 \mathrm{~cm}$ in the frequency range of $\sim 100 \mathrm{~Hz}$ to $\sim 400 \mathrm{~Hz}$, while anisotropic neutrino emission is responsible for a superimposed low-frequency evolution of the wave amplitude, which thus can grow to maximum values of $10-20 \mathrm{~cm}$. Variations of the mass-quadrupole moment caused by convective activity inside the nascent neutron star contribute a highfrequency component $(300-600 \mathrm{~Hz})$ to the $\mathrm{GW}$ signal during the post-accretion phase. The GW signals exhibit strong variability between the two polarizations, different explosion simulations and different observer directions, and besides common basic features do not possess any template character.

Finally we would like to reflect on some of the deficiencies of the presented 3D models. Because of the ray-by-ray treatment of the $v$-transport, the directional variations of the neutrino emission in response to local inhomogeneities in the star may be overestimated (Ott et al. 2008; Brandt et al. 2011). However, when we evaluate observable signals, these artificial effects are mostly compensated for by integrations of the neutrino flux densities over the surface areas visible to observers from different viewing directions (see Eqs. (5), (7), (10), (11)), or by the integration of the neutrino energy loss in all directions (Eqs. (13), (53)).

Another deficiency concerns the usage of the inner radial grid boundary, because of which our simulations do not fully include (either in space or time) the convective flow occurring in the PNS interior after core bounce (Keil et al 1996; Buras et al. 2006; Dessart et al. 2006). Moreover, convective activity in the simulated outer layers of the PNS occurs for special conditions: it is triggered only when the artificially imposed inflow of neutrino energy and lepton number through the inner radial boundary into the adjacent layers is faster than the neutrinos can carry away this energy or lepton number. Whether this is the case sensitively depends on the employed neutrino-transport approximation, but also on the location and contraction of the grid boundary, the chosen values of the boundary luminosities, and on the stellar progenitor. Its mass-infall rate decides how much mass accumulates in the near-surface layers of the PNS outside the inner grid boundary. Because the position of and the conditions imposed at the inner boundary can thus influence the neutrino emission properties, in particular during the post-accretion phase, our respective model predictions must be considered with care. While they do not allow us to make any definite statements concerning the detailed neutrino signal of a particular progenitor model due to the neglected treatment of the inner parts of the proto-neutron star, the models nevertheless show that convective flows below the neutrinosphere are likely to imprint themselves on the neutrino emission, and hence also on the GW signal of core-collapse supernovae. A measurement of these signals may actually provide some insight into the conditions inside protoneutron stars.

Acknowledgements. This work was supported by the Deutsche Forschungsgemeinschaft through the Transregional Collaborative Research Centers SFB/TR 27 "Neutrinos and Beyond" and SFB/TR 7 "Gravitational Wave Astronomy" and the Cluster of Excellence EXC 153 "Origin and Structure of the Universe" (http: //www . universe-cluster.de).

\section{References}

Arcones, A., Janka, H.-T., \& Scheck, L. 2007, A\&A, 467, 1227 Baade, W., \& Zwicky, F. 1934, Phys. Rev., 45, 138

Blanchet, L., Damour, T., \& Schäfer, G. 1990, MNRAS, 242, 289

Brandt, T. D., Burrows, A., Ott, C. D., \& Livne, E. 2011, ApJ, 728, 8

Buras, R., Janka, H.-Th., Rampp, M., \& Kifonidis, K. 2006, A\&A, 457, 281

Burrows, A., \& Hayes, J. 1996, Phys. Rev. Lett., 76, 352

Burrows, A., Hayes, J., \& Fryxell, B. A. 1995, ApJ, 450, 830

Cerda-Duran, P., Faye, G., Dimmelmeier, H., et al. 2005, A\&A, 439, 1033

Colella, P., \& Glaz, H. M. 1985, J. Chem. Phys., 59, 264

Collela, P., \& Woodward, P. R. 1984, J. Chem. Phys., 54, 174

Dessart, L., Burrows, A., Livne, E., \& Ott, C. D. 2006, ApJ, 645, 534

Dimmelmeier, H., Font, J. A., \& Müller, E. 2001, ApJ, 560, L163

Dimmelmeier, H., Font, J. A., \& Müller, E. 2002, A\&A, 393, 523

Dimmelmeier, H., Ott, C. D., Janka, H.-T., Marek, A., \& Müller, E. 2007, Phys. Rev. Lett., 98, 01101

Dimmelmeier, H., Ott, C. D., Marek, A., \& Janka, H.-T. 2008, Phys. Rev. D, 78, 064056

Epstein, R. 1978, ApJ, 223, 1037

Finn, L. S., \& Evans, C. R. 1990, ApJ, 351, 588

Fryer, C. L. 2004, ApJ, 601, L175

Fryer, C. L., \& New, K. C. B. 2011, Liv. Rev. Rel., 14, 1, http://www. livingreviews .org/lrr-2011-1

Fryer, C. L., Holz, D. E., \& Hughes, S. A. 2004, ApJ, 609, 288

Fryxell, B., Müller, E., \& Arnett, D. 1991, ApJ, 367, 619

Heger, A., Woosley, S. E., \& Spruit, H. C. 2005, ApJ, 626, 350

Janka, H.-T. 1991, A\&A, 244, 378

Janka, H.-T., \& Mönchmeyer, R. 1989a, A\&A, 209, L5

Janka, H.-T., \& Mönchmeyer, R. 1989b, A\&A, 226, 69

Janka, H.-T., \& Müller, E. 1996, A\&A, 306, 167

Kageyama, A., \& Sato, T. 2004, Geochem. Geophys. Geosys., 5, Q09005

Keil, W., Janka, H.-T., \& Müller, E. 1996, ApJ, 473, L111

Kotake, K., Yamada, S., \& Sato, K. 2003, Phys. Rev. D, 68, 44023

Kotake, K., Yamada, S., Sato, K., et al. 2004, Phys. Rev. D, 69, 124004

Kotake, K., Yamada, S., \& Sato, K. 2005, ApJ, 618, 474

Kotake, K., Sato, K., \& Takahashi, K. 2006, Rep. Prog. Phys., 69, 971

Kotake, K., Onishi, N., \& Yamada, S. 2007, ApJ, 655, 406

Kotake, K., Iwakami, W., Ohnishi, N., \& Yamada, S. 2009a, ApJ, 704, 951

Kotake, K., Iwakami, W., Ohnishi, N., \& Yamada, S. 2009b, ApJ, 697, L133

Kotake, K., Iwakami Nakano, W., \& Ohnishi, N. 2011, ApJ, 736, 124

Liebendörfer, M. 2005, ApJ, 633, 1042

Liebendörfer, M., Whitehouse, S. C., \& Fischer, T. 2009, ApJ, 698, 1174

Limongi, M., Straniero, O., \& Chieffi, A. 2000, ApJS, 129, 625

Liou, M. S. 1996, J. Chem. Phys., 129, 364

Lund, T., Marek, A., Lunardini, C., Janka, H.-Th. \& Raffelt, G. 2010, Phys. Rev. D, 82, 063007

Marek, A., \& Janka, H.-Th. 2009, ApJ, 694, 664 
Marek, A., Dimmelmeier, H., Janka, H.-Th., Müller, E., \& Buras, R. 2006, A\&A, 445,273

Marek, A., Janka, H.-Th., \& Müller, E. 2009, A\&A, 496, 475

Mihalas, D. 1978, Stellar Atmospheres (San Francisco, USA: Freeman)

Misner, C. W., Thorne, K. S., \& Wheeler, J. A. 1973, Gravitation, Chap. 35 (San Francisco, USA: Freeman)

Mönchmeyer, R., Schäfer, G., Müller, E., \& Kates, R. 1991, A\&A, 246, 417

Morse, P. M., \& Feshbach, H. 1953, Methods of Theoretical Physics (New York, USA: McGraw-Hill)

Müller, E. 1982, A\&A, 114, 53

Müller, E., \& Janka, H.-Th. 1997, A\&A, 317, 140

Müller, E., \& Steinmetz, M. 1995, Comp. Phys. Comm., 89, 45

Müller, E., Fryxell, B., \& Arnett, D. 1991a, A\&A, 251, 505

Müller, E., Fryxell, B., \& Arnett, D. 1991b, in European Southern Observatory Conference and Workshop Proceedings, ed. I. J. Danziger, \& K. Kjaer, 37, 99

Müller, E., Rampp, M., Buras, R., Janka, H.-Th., \& Shoemaker, D. H. 2004, ApJ, 603, 221

Müller, B., Dimmelmeier, H., \& Müller, E. 2008, A\&A, 489, 301

Murphy, J. W., Ott, C. D., \& Burrows, A. 2009, ApJ, 707, 1173

Nakamura, T., \& Oohara, K. 1989, in Frontiers in Numerical Relativity, ed. C. R. Evans, L. S. Finn, \& D. W. Hobill (Cambridge Univ. Press), 254 Obergaulinger, M., Aloy, M. A., \& Müller, E. 2006a, A\&A, 450, 1107

Obergaulinger, M., Aloy, M. A., \& Müller, E. 2006b, A\&A, 457, 209

Oohara, K., Nakamura, T., \& Shibata, M. 1997, Prog. Theor. Phys. Suppl., 128, 183

Ott, C. D. 2009, Class. Quant. Grav., 26, 063001

Ott, C. D., Burrows, A., Livne, E., \& Walder, R. 2004, ApJ, 600, 834

Ott, C. D., Burrows, A., Dessart, L., \& Livne, E. 2006, Phys. Rev. Lett., 96, 201102
Ott, C. D., Dimmelmeier, H., Marek, A., et al. 2007, Phys. Rev. Lett., 98, 261101 Ott, C. D., Burrows, A., Dessart, L., \& Livne, E. 2008, ApJ, 685, 1069

Ott, C. D., Reisswig, C., Schnetter, E., et al. 2011, Phys. Rev. Lett., 106, 161103

Pejcha, O., \& Thompson, T. A. 2012, ApJ, accepted [arXiv: 1103.4864]

Plewa, T., \& Müller, E. 1999, A\&A, 342, 179

Quirk, J. J. 1994, Int. J. Num. Meth. Fluids, 18, 555

Rampp, M., \& Janka, H.-T. 2002, A\&A, 396, 361

Rampp, M., Müller, E., \& Ruffert, M. 1998, A\&A, 332, 969

Saijo, M. 2006, Phys. Rev. D, 71, 104038

Scheck, L., Kifonidis, K., Janka, H.-T., \& Müller, E. 2006, A\&A, 457, 963

Scheck, L., Janka, H.-T., Foglizzo, T., \& Kifonidis, K. 2008, A\&A, 477, 931

Scheidegger, S., Fischer, T., Whitehouse, S. C., \& Liebendörfer, M. 2008, A\&A, 490, 231

Scheidegger, S., Käppeli, R., Whitehouse, S. C., Fischer, T., \& Liebendörfer, M. 2010, A\&A, 514, A51

Shibata, M. 2003, Phys. Rev. D, 67, 024033

Shibata, M., \& Sekiguchi, Y. 2004, Phys. Rev. D, 69, 084024

Shibata, M., \& Sekiguchi, Y. 2005, Phys. Rev. D, 71, 024014

Shigeyama, T., \& Nomoto, N. 1990, ApJ, 360, 242

Strang, G. 1968, SIAM J. Numer. Anal., 5, 506

Wongwathanarat, A. 2011, Ph.D. Thesis, Technical University Munich Wongwathanarat, A., Hammer, N. J., \& Müller, E. 2010a, A\&A, 514, A48

Wongwathanarat, A., Janka, H.-T., \& Müller, E. 2010b, ApJ, 725, L106

Woosley, S. E., \& Weaver, T. A. 1995, ApJS, 101, 181

Yakunin, K. N., Marronetti, P., Mezzacappa, A., et al. 2010, Class. Quant. Grav., 27, 194005

Yamada, S., \& Sato, K. 1994, ApJ, 450, 245

Yamada, S., \& Sawai, H. 2004, ApJ, 608, 907

Zwerger, T., \& Müller, E. 1997, A\&A, 320, 209 